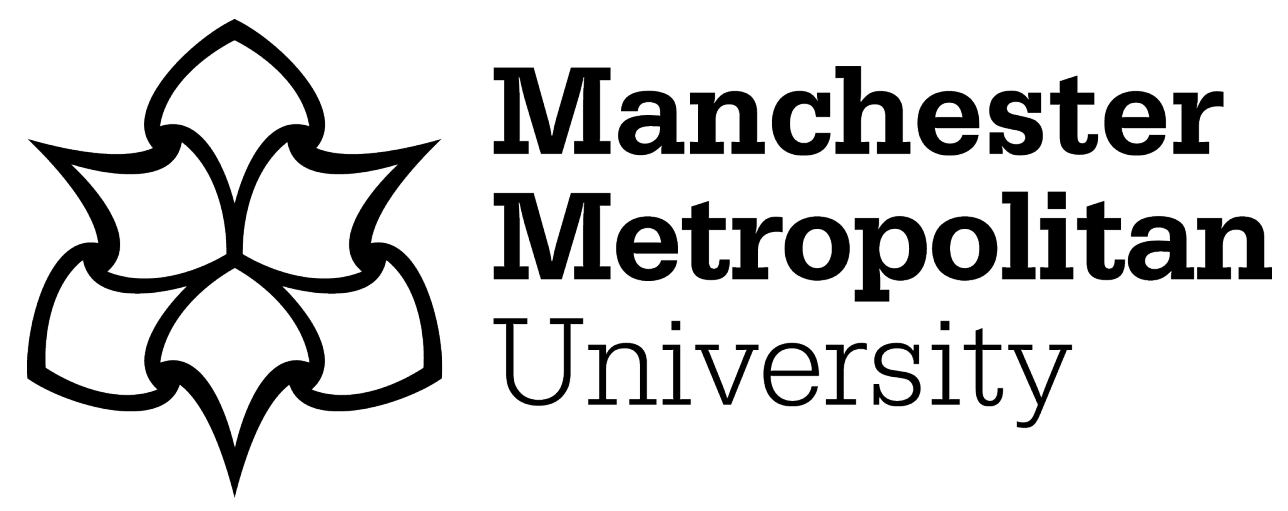

Hubert, McKenzie A, Patel, Anjli M, Gallo, Alessandro, Liu, Yunzhi, Valle, Eduardo, Ben-Naim, Micha, Sanchez, Joel, Sokaras, Dimosthenis, Sinclair, Robert, Nørskov, Jens K, King, Laurie A, Bajdich, Michal and Jaramillo, Thomas F (2020) Acidic Oxygen Evolution Reaction Activity-Stability Relationships in Ru-Based Pyrochlores. ACS Catalysis, 10 (20). pp. 1218212196. ISSN 2155-5435

Downloaded from: https://e-space.mmu.ac.uk/626603/

Version: Accepted Version

Publisher: American Chemical Society (ACS)

DOI: https://doi.org/10.1021/acscatal.0c02252

Please cite the published version 


\section{Acidic oxygen evolution reaction activity-stability relationships in Ru-based pyrochlores}

McKenzie A. Hubert, ${ }^{\dagger}, \#$ Anjli M. Patel, ${ }^{\dagger, \#}$ Alessandro Gallo, ${ }^{\ddagger}$ Yunzhi Liu, Eduardo Valle, ${ }^{\dagger}$ Micha Ben-Naim, ${ }^{\dagger}$ Joel Sanchez, ${ }^{\dagger}$ Dimosthenis Sokaras, ${ }^{\S}$ Robert Sinclair, ${ }^{*} \llbracket$ Jens K. Nørskov, $\|$ Laurie A. King, ${ }^{*, \perp}$ Michal Bajdich, ${ }^{*, \ddagger}$ and Thomas F. Jaramillo*, $\dagger$

$\dagger$ †UNCAT Center for Interface Science and Catalysis, Department of Chemical Engineering, Stanford University, Stanford, CA

$\ddagger S U N C A T$ Center for Interface Science and Catalysis, SLAC National Accelerator Laboratory, Menlo Park, CA

\Department of Materials Science and Engineering, Stanford University, Stanford, CA $\S S L A C$ National Accelerator Laboratory, Menlo Park, CA

||Department of Physics, Technical University of Denmark, 2800 Kongens Lyngby,

Denmark

$\perp$ Faculty of Science and Engineering, Manchester Metropolitan University, Manchester, M1 $5 G D, U K$ \# These authors contributed equally to this work.

E-mail: bobsinc@stanford.edu; I.king@mmu.ac.uk; bajdich@slac.stanford.edu; jaramillo@stanford.edu 


\begin{abstract}
Ru-based oxygen evolution reaction (OER) catalysts show significant promise for efficient water electrolysis, but rapid degradation poses a major challenge for commercial applications. In this work, we explore several Ru-based pyrochlores $\left(\mathrm{A}_{2} \mathrm{Ru}_{2} \mathrm{O}_{7}, \mathrm{~A}\right.$ $=\mathrm{Y}, \mathrm{Nd}, \mathrm{Gd}, \mathrm{Bi})$ as OER catalysts and demonstrate improved activity and stability of catalytic $\mathrm{Ru}$ sites relative to $\mathrm{RuO}_{2}$. Furthermore, we combine complementary experimental and theoretical analysis to understand how the A-site element impacts activity and stability under acidic OER conditions. Amongst the $\mathrm{A}_{2} \mathrm{Ru}_{2} \mathrm{O}_{7}$ studied herein, we find that a longer $\mathrm{Ru}-\mathrm{O}$ bond and a weaker interaction of the $\mathrm{Ru} 4 d$ and $\mathrm{O} 2 p$ orbitals compared to $\mathrm{RuO}_{2}$ results in enhanced initial activity. We observe that the OER activity of the catalysts changes over time and is accompanied by both A-site and $\mathrm{Ru}$ dissolution at different relative rates depending on the identity of the A-site. Pourbaix diagrams constructed using density functional theory (DFT) calculations reveal a driving force for this experimentally observed dissolution, indicating that all compositions studied herein are thermodynamically unstable in acidic OER conditions. Theoretical activity predictions show consistent trends between A-site cation leaching and OER activity. These trends coupled with Bader charge analysis suggest that dissolution exposes highly oxidized $\mathrm{Ru}$ sites that exhibit enhanced activity. Overall, using the stability number $\left(\mathrm{mol}_{\mathrm{O}_{2} \text { evolved }} / \mathrm{mol}_{\mathrm{Ru} \text { dissolved }}\right)$ as a comparative metric, the $\mathrm{A}_{2} \mathrm{Ru}_{2} \mathrm{O}_{7}$ materials studied in this work show substantially greater stability than a standard $\mathrm{RuO}_{2}$ and commensurate stability to some Ir mixed metal oxides. The insights described herein provide a path to further enhance Ru catalyst activity and durability, ultimately improving the efficiency of water electrolyzers.
\end{abstract}

\title{
Keywords
}

water splitting, oxygen evolution, ruthenium, pyrochlore, theoretical Pourbaix stability, dissolution, activity descriptors 


\section{Introduction}

Seasonal variation in wind and solar power production creates an urgent need for long-term and terawatt (TW)-scale energy storage. ${ }^{1}$ One promising solution is to store renewable energy in the form of hydrogen produced via water electrolysis. Specifically, proton exchange membrane (PEM) electrolyzers can achieve high current densities with a compact design and fast responses to dynamic power supply, making them attractive for TW-scale energy storage. However, substantial operating costs resulting from inefficient catalysts impede widespread adoption of this technology. ${ }^{2,3}$ In particular, the anode catalyst requires a large overpotential to overcome sluggish kinetics for the oxygen evolution reaction (OER). This highly oxidizing voltage coupled with the acidic nature of the PEM creates a corrosive environment that is particularly detrimental to the stability of the OER catalyst. ${ }^{4}$ As a result, very few OER catalyst candidates are both active and stable under the harsh, acidic, oxidizing conditions relevant for reliable operation of PEM electrolyzers. ${ }^{5}$ The lack of active and stable catalysts under these conditions presents a great scientific and technological challenge that must be overcome to improve the economic viability of PEM electrolyzers for industrial-scale renewable hydrogen production. ${ }^{6}$

Researchers have evaluated both non-precious and precious metal oxides for PEM OER catalysis. While a few non-precious metal catalysts have demonstrated some stability and activity in acidic electrolyte, the activity of these catalysts does not rival that of precious metals. $^{7,8}$ Indeed, $\mathrm{IrO}_{\mathrm{x}}$ remains the state-of-the-art catalyst for the OER owing to its ability to maintain relatively high activity with minimal degradation. ${ }^{5}$ Other precious metals, such as $\mathrm{Ru}$, demonstrate greater intrinsic activity relative to Ir, but they suffer from greater dissolution during OER. ${ }^{9}$ One strategy to reduce operating costs of PEM electrolyzers is to improve the stability of Ru-based catalysts by using mixed metal oxide compositions.

Mixed metal oxides of varying crystal structures, such as pyrochlores $\left(\mathrm{A}_{2} \mathrm{~B}_{2} \mathrm{O}_{7}\right)$ and perovskites $\left(\mathrm{ABO}_{3}\right)$, have shown promising enhancements for acidic OER activity and stability

beyond that of pure precious metal oxides. ${ }^{10-19}$ In these mixed metal oxides $\left(\mathrm{AB}_{\mathrm{x}} \mathrm{O}_{\mathrm{y}}\right)$, the 
A-site is a non-precious metal while the B-site is typically an active precious metal like Ir or Ru. The A-site and B-site elements must have compatible ionic radii to form the desired crystal structure, restricting the number of possible compositions. ${ }^{20}$

Studies have shown that varying the $\mathrm{A}$-site element in $\mathrm{AB}_{\mathrm{x}} \mathrm{O}_{\mathrm{y}}(\mathrm{B}=\mathrm{Ir}, \mathrm{Ru})$ can tune OER performance; ${ }^{10-12}$ however, the explanation for enhanced activity remains unclear. It is widely accepted that the precious metal B-site rather than the A-site element in these structures are active for OER. ${ }^{13,19}$ Activity enhancements of $\mathrm{A}_{2} \mathrm{~B}_{2} \mathrm{O}_{7}$ and $\mathrm{ABO}_{3}(\mathrm{~B}=\mathrm{Ir}, \mathrm{Ru})$ over $\mathrm{RuO}_{\mathrm{x}}$ or $\mathrm{IrO}_{\mathrm{x}}$ have been attributed to a multitude of factors: changing the B-O-B bond angle ${ }^{11}$ or lattice parameter, ${ }^{12}$ hybridizing B-site $d$ states with O $2 p$ states, ${ }^{11,16,21}$ shifting the d-band center, ${ }^{22}$ and dissolving the A-site to form an amorphous $\mathrm{BO}_{\mathrm{x}}$ structure during catalysis. ${ }^{10,13}$ From a theoretical perspective in particular, previous reports have linked the electronic structure of pyrochlores to their experimentally observed OER activity. ${ }^{16,17}$ The field would benefit from a more comprehensive understanding of the catalytic activity of these systems from a mechanistic perspective via OER intermediate adsorption energetics, and greater theoretical insight into the origin of enhanced performance of pyrochlores would advance the field.

Furthermore, the stability of these materials under acidic OER conditions, and specifically the extent of A- and B-site dissolution, varies widely across studies, which may arise from non-standardized electrochemical methods used to assess catalyst stability. In some reports, no A-site dissolution is reported or detected, ${ }^{11}$ while in others significant amounts of A-site dissolution (in some cases $>50 \%$ ) is detected. ${ }^{10,12,13,16-18}$ Interestingly, B-site dissolution is either not reported or detected for most Ir-based mixed metal oxide catalysts, ${ }^{10-13}$ while B-site dissolution is detected for Ru-based mixed metal oxides. ${ }^{12,17,18,23}$ Collectively, the wide range of reported stability results indicates a gap in fundamental understanding of the thermodynamic and kinetic driving forces that govern dissolution of $\mathrm{AB}_{\mathrm{x}} \mathrm{O}_{\mathrm{y}}(\mathrm{B}=\mathrm{Ir}$, $\mathrm{Ru}$ ) materials in acid during OER catalysis. Further, few reports discuss how dissolution processes may influence activity or translate measured dissolution results into a metric that 
can be compared across catalyst materials.

In this work, we combine experimental techniques and theoretical models to study the influence of the A-site element on the OER activity and stability in acidic electrolyte of several Ru-based pyrochlores $\left(\mathrm{A}_{2} \mathrm{Ru}_{2} \mathrm{O}_{7}, \mathrm{~A}=\mathrm{Y}, \mathrm{Nd}, \mathrm{Gd}, \mathrm{Bi}\right)$. We selected this unique set of A-site elements to form $\mathrm{A}_{2} \mathrm{Ru}_{2} \mathrm{O}_{7}$ phases with varying physical and electronic properties such that we could investigate a wide range of both structural and electronic impacts on OER activity and stability. First, we synthesize highly crystalline Ru pyrochlore powders and characterize them to determine their composition and morphology. Then, we compare their experimental OER activity and stability to a commercial $\mathrm{RuO}_{2}$ standard to observe the influences of composition and structure on performance. Finally, we build on these findings using theoretical insights from Pourbaix analysis and DFT-predicted OER activity trends resulting from A-site leaching. Our experimental and theoretical results highlight the inherent relationship between activity and stability in these systems and reflect the importance of monitoring catalyst dissolution while measuring OER performance.

\section{Experimental Methods}

Materials. All chemicals and materials were used as received. Yttrium nitrate hydrate $\left(\mathrm{Y}\left(\mathrm{NO}_{3}\right)_{3} \cdot \mathrm{xH}_{2} \mathrm{O}, \quad 99.9 \%\right.$, Spectrum Chemicals), bismuth nitrate pentahydrate $\left(\mathrm{Bi}\left(\mathrm{NO}_{3}\right)_{3} \cdot 5 \mathrm{H}_{2} \mathrm{O}, 99.99 \%\right.$, Acros Organics $)$, gadolinium nitrate hydrate $\left(\mathrm{Gd}\left(\mathrm{NO}_{3}\right)_{3} \cdot \mathrm{xH}_{2} \mathrm{O}\right.$, 99.99\%, Alfa Aesar), neodymium nitrate hydrate $\left(\mathrm{Nd}\left(\mathrm{NO}_{3}\right)_{3} \cdot \mathrm{xH}_{2} \mathrm{O}, 99.99 \%\right.$, Alfa Aesar), and ruthenium nitrosyl nitrate solution $\left(\mathrm{RuNO}\left(\mathrm{NO}_{3}\right)_{3}, 1.5 \% \mathrm{Ru}\right.$ in dilute nitric acid, Strem Chemicals) were used as catalyst precursors. Ruthenium oxide $\left(\mathrm{RuO}_{2}, 99.9 \%\right.$, Aldrich) was used as a standard for comparison. Citric acid $\left(\mathrm{C}_{6} \mathrm{H}_{8} \mathrm{O}_{7}\right.$, crystalline, 99.5\%, Alfa Aesar) was used as a chelating agent during catalyst synthesis. Vulcan carbon (Vulcan XC 72, Fuel Cell Store) was used as the catalyst support during electrochemical testing. IPA (99.99\%, HPLC grade, Fisher Chemical) was used as the catalyst ink solvent, and Nafion perfluorinated resin 
(10 wt\% in $\mathrm{H}_{2} \mathrm{O}$, Sigma-Aldrich) was used as a binder in the catalyst ink. All catalyst inks were tested on polished glassy carbon disks or gold disks ( $5 \mathrm{~mm}$ diameter). The electrolyte (0.1 $\left.\mathrm{M} \mathrm{HClO}_{4}\right)$ was prepared from concentrated perchloric acid (70\% concentration, double distilled, GFS Chemicals). A graphite rod ( $<2$ ppm impurities, Ted Pella) was used as the counter electrode and the electrolyte was continuously purged with oxygen gas $(99.993 \%$ purity) during electrochemical tests.

$\mathbf{A}_{2} \mathbf{R u}_{2} \mathrm{O}_{7}$ Synthesis. The $\mathrm{A}_{2} \mathrm{Ru}_{2} \mathrm{O}_{7}$ catalysts were prepared via a sol-gel method adapted from Ref. 16. In brief, the A-site metal nitrate hydrate (0.5 mmol A-site) and citric acid $\left(0.42 \mathrm{~g}, \sim 4.5\right.$ mole $\left._{\text {citric acid }} / \mathrm{mole}_{\mathrm{A}-\text { site }}\right)$ were dissolved in $10 \mathrm{~mL}$ of Millipore water. This solution was sonicated for $10 \mathrm{~min}$ to fully dissolve the citric acid and metal nitrate. Then, while stirring (300 rpm), ruthenium nitrosyl nitrate solution (1.5 wt\% Ru) was added in an equal molar amount to the A-site cation (0.5 mmol Ru, $3.09 \mathrm{~mL})$. This solution was heated to $80^{\circ} \mathrm{C}$ while continuously stirring until the solvent fully evaporated $(4-6 \mathrm{hr})$. The resulting rust-colored gel was further dried at $\sim 90^{\circ} \mathrm{C}$ overnight in a drying oven. The solid was subsequently ground with a mortar and pestle (1-2 min) to create a fine powder. This powder was then transferred to a zirconia combustion boat and calcined in air at $1000^{\circ} \mathrm{C}$ (Y, Nd, Gd samples) or $700^{\circ} \mathrm{C}$ (Bi sample) for $6 \mathrm{hr}$ to form the pyrochlore phase. A lower calcination temperature for the Bi sample was required to achieve a pyrochlore structure with a BET surface area similar to the other catalysts. A heating ramp rate of $2^{\circ} \mathrm{C} / \mathrm{min}$ was used for all samples. The catalyst was cooled to $<100^{\circ} \mathrm{C}(6-10 \mathrm{hr})$ before removing from the furnace. Finally, the resulting catalysts were again ground with a mortar and pestle (1-2 $\min )$.

Ex-situ Physical Characterization. X-ray diffraction (XRD) of the as-prepared pyrochlores was performed using a Bruker D8 Venture with Mo $\mathrm{K}_{\alpha}$ radiation to determine crystallinity. XRD of the pyrochlores after electrochemical testing was performed using a PANanalytical XPert with $\mathrm{Cu} \mathrm{K}_{\alpha}$ radiation at a grazing incidence angle to enhance the catalyst signal relative to the substrate. Diffraction patterns from the different radiation 
sources were compared using Bragg's law.

X-ray photoelectron spectroscopy (XPS) was performed on a PHI Versaprobe 3 with Al $\mathrm{K}_{\alpha}$ radiation to determine composition and elemental oxidation state. Powder samples were mounted onto the sample holder using double-sided $\mathrm{Cu}$ tape to minimize carbon signal. All XPS data sets were analyzed using CasaXPS software. The surface A:Ru molar ratio was determined by fitting the Ru $3 d$ and A-site ( $\mathrm{Y} 3 d, \mathrm{Nd} 4 d$, Gd 4d, Bi 4f) regions from high resolution scans, then normalizing peak area by the relative sensitivity factors. Note that the $\mathrm{Ru} 3 d$ region overlaps with the $\mathrm{C} 1 s$ signal arising from adventitious carbon. $\mathrm{Ru} 3 d$ peaks were fit by applying constraints on the $3 d_{3 / 2}$ peak area (x $\left.2 / 3\right)$, full width half max ( $\mathrm{x}$ 1 ), and position $(+4.17 \mathrm{eV})$ relative to the $3 d_{5 / 2}$ peak and using an asymmetric lineshape typical of $\mathrm{RuO}_{2} \cdot{ }^{24}$ After fitting the $\mathrm{Ru} 3 d$ peaks, the residual signal was attributed to a single C $1 s$ peak. All high resolution scans were shifted to align the $\mathrm{C}$ 1s peak to $284.8 \mathrm{eV}$.

BET (Brunauer, Emmett, and Teller) surface area measurements were conducted using a Quantachrome Autoflow BET+. Scanning electron microscopy (SEM) was performed using a FEI Magellan 400 XHR to determine catalyst morphology. Auger electron spectroscopy (AES) was performed on a PHI 700 to map catalyst and carbon distribution on the substrate. Scanning transmission electron microscopy with energy dispersive x-ray spectroscopy (STEM-EDS) and STEM annular dark field (STEM-ADF) was performed using a FEI Ti$\tan 80-300$ with a probe size of $0.3 \mathrm{~nm}$ to map elemental composition of the catalysts. The STEM-ADF images were taken with convergence angles of 18.6-25.4 mrad. Samples were prepared for TEM by sonicating the catalyst ink-coated substrates in IPA for $1 \mathrm{~min}$, then depositing 2 drops of the IPA onto TEM grids.

All x-ray absorption spectroscopy (XAS) was performed at the Stanford Synchrotron Radiation Lightsource (SSRL) at SLAC National Laboratory. Ex-situ measurements of the catalyst powders at the O K-edge $(\sim 0.5 \mathrm{keV})$ were collected at beamline $8-2$ in total electron yield (tey) mode averaging 5-10 scans. Ex-situ x-ray absorption near edge structure (XANES) and extended x-ray absorption fine structure (EXAFS) measurements at the Ru 
K-edge $(\sim 22.1 \mathrm{keV})$ were collected for the powder catalysts at ambient temperature and pressure in fluorescence mode at beamline 9-3 averaging 3-4 scans. XAS analysis was performed using Athena and Artemis software. ${ }^{25}$ Details of the fit parameters and simulations can be found in the supporting information.

Electrochemical Characterization. Catalyst inks were prepared by dispersing the catalyst $(2 \mathrm{mg})$ and Vulcan carbon $(1 \mathrm{mg})$ in a solution of IPA $(2 \mathrm{~mL})$ and Nafion $(0.6 \mu \mathrm{L})$. For control measurements, inks only containing Vulcan carbon (no catalyst) were prepared with IPA $(2 \mathrm{~mL})$ and Nafion $(0.6 \mu \mathrm{L})$. All inks were sonicated $(1 \mathrm{hr})$ immediately before electrochemical testing. Electrodes were prepared by drop casting the catalyst ink onto a substrate and rotating the electrode $(\sim 250 \mathrm{rpm})$ until the surface was visibly dry $(\sim 5 \mathrm{~min})$. The OER performance of each catalyst was assessed in $\mathrm{O}_{2}$-purged $0.1 \mathrm{M} \mathrm{HClO}_{4}$ electrolyte $(50 \mathrm{~mL})$ using a three-electrode configuration with a graphite counter electrode and $\mathrm{Ag}-\mathrm{AgCl}$ reference electrode. A rotating disk electrode (RDE) configuration and BioLogic VMP3 potentiostat were used for all electrochemical tests unless otherwise noted.

Electrodes for activity measurements were prepared by depositing $5 \mu \mathrm{L}$ of catalyst ink onto a glassy carbon disk substrate $\left(0.196 \mathrm{~cm}^{2}\right)$ resulting in a catalyst loading of $\sim 25$ $\mu \mathrm{g} / \mathrm{cm}^{2}$ geo. The OER activity was assessed using cyclic voltammetry $(\mathrm{CV})$ where the potential was cycled between $1.35 \mathrm{~V}$ and $\sim 1.6 \mathrm{~V}$ vs. RHE (the potential required to reach a current density of $\sim 10 \mathrm{~mA} / \mathrm{cm}^{2}$ geo ) at a scan rate of $10 \mathrm{mV} / \mathrm{s}$. This scan rate is within the range of scan rates recommended in OER catalyst benchmarking protocols ${ }^{26}$ and used by others in the field. ${ }^{12}$ Impedance measurements were conducted at open circuit potential prior to CV measurements and all measurements were corrected for the Ohmic resistance of the electrolyte after testing ( $0 \%$ compensation during testing). At least 3 independent measurements were collected for each catalyst to account for variations caused by slight differences in catalyst dispersion or mass loading.

Electrodes for stability measurements were prepared by depositing $15 \mu \mathrm{L}$ of catalyst ink onto a gold disk substrate $\left(0.196 \mathrm{~cm}^{2}\right)$ resulting in a catalyst loading of $\sim 75 \mu \mathrm{g} / \mathrm{cm}^{2}$ geo 
Gold substrates have demonstrated greater resistance to degradation during OER testing in acid than glassy carbon disks and therefore mitigate substrate degradation when evaluating system degradation mechanisms and quantifying catalyst dissolution. ${ }^{27,28}$ Catalyst stability was determined using chronoamperometry (CA). The applied voltage was corrected for the Ohmic resistance of the electrolyte (85\%) during the experiment using impedance measurements. Following an initial impedance measurement, the voltage was increased to $1.56 \mathrm{~V}$ vs. RHE at $10 \mathrm{mV} / \mathrm{s}$ and subsequently held at that potential for $10 \mathrm{hr}$.

Electrolyte Characterization. Inductively coupled plasma mass spectrometry (ICPMS) was performed using a Thermo Scientific XSERIES 2 to quantify the amount of dissolved catalyst in the electrolyte. Electrolyte aliquots $(4 \mathrm{~mL})$ were taken after 3, 6, and $10 \mathrm{hr}$ of CA testing for ICP-MS analysis and fresh electrolyte $(4 \mathrm{~mL})$ was replenished such that the total volume of electrolyte $(50 \mathrm{~mL})$ was unchanged. Millipore water $(4 \mathrm{~mL})$ was added to each aliquot to achieve the minimum volume required for ICP-MS analysis. ICP-MS calibration standards were prepared using serial dilutions of commercial stock solutions. Quality control samples were prepared independently using the commercial stock solutions. The lowest reliable quantification limit for the dissolved mass fraction of each element was determined based on the catalyst loading, electrolyte volume, standard calibration curves, and baseline ion counts in solution: $\sim 1 \%$ for $\mathrm{Ru}, \mathrm{Y}, \mathrm{Nd}, \mathrm{Gd}$ and $\sim 2 \%$ for $\mathrm{Bi}$. In other words, we were able to reliably quantify dissolution if $>1 \%$ of the total $\mathrm{Ru}$ in the catalyst dissolved into the electrolyte, for example. A sample calculation for these limits can be found in the supporting information.

In-situ X-ray Absorbance Spectroscopy. In-situ XANES and EXAFS measurements at the $\mathrm{Ru} \mathrm{K}$-edge $(\sim 22.1 \mathrm{keV})$ were conducted at SSRL's beamline 9-3. Samples were prepared by first pyrolyzing the catalyst substrate, a Kapton film (0.002 in. thick) between two graphite plates at $1000^{\circ} \mathrm{C}$ for $1 \mathrm{hr}$ in $\mathrm{N}_{2}$. The $\mathrm{Y}_{2} \mathrm{Ru}_{2} \mathrm{O}_{7}$ catalyst ink was then deposited onto the pyrolyzed Kapton film substrate $\left(50 \mu \mathrm{g}_{\mathrm{Ru}} / \mathrm{cm}^{2}\right)$, which was epoxied over a hole $(0.67 \mathrm{~cm}$ diameter $)$ in a high-density polyethylene bottle. The deposited catalyst was 
in contact with $\mathrm{O}_{2}$-sparged $0.1 \mathrm{M} \mathrm{HClO}_{4}$ electrolyte in the bottle, while the back of the film was exposed to air and contacted with $\mathrm{Cu}$ tape. Electrochemical data was recorded with a Biologic SP-300 potentiostat. Electrochemical performance was assessed using a 3electrode configuration with a graphite counter electrode and $\mathrm{Hg}-\mathrm{HgSO}_{4}$ reference electrode inserted through the bottle cap. This experimental setup was largely modelled after Ref. 29 , where schematics of the setup can be found. X-rays penetrated the catalyst through the pyrolyzed Kapton film substrate, and x-ray fluorescence was collected using a Lytle detector with Mo filter. XAS measurements were collected at open circuit voltage, $1.27 \mathrm{~V}, 1.47 \mathrm{~V}$, and $1.58 \mathrm{~V}$ vs. RHE at ambient temperature and pressure. XAS analysis was performed using Athena and Artemis software averaging 3-4 scans. ${ }^{25}$ The electrolyte was collected after the experiment for ICP-MS analysis.

Computational Methods. DFT computations of the pyrochlore structures were conducted using the PerdewBurke-Ernzerhof (PBE) functional ${ }^{30}$ in the Vienna Ab initio Simulation Package (VASP) ${ }^{31-33}$ with projector augmented wave method (PAW) potentials ${ }^{34}$ and spin polarization. For pyrochlore bulk structures, a plane-wave energy cutoff of $600 \mathrm{eV}$ was used with a $4 \times 4 \times 4$ Monkhorst-Pack k-point grid, ${ }^{35}$ from which bulk Pourbaix diagrams were constructed using the Pymatgen software. ${ }^{36}$ Formation energies of solid species were computed by DFT and corrected for compatibility with experimental values for aqueous species, including ions. Additional details on free energy corrections are provided in the "Formation Energy Computation for Pourbaix Analysis" section of the supporting information.

For pyrochlore surface calculations, a plane-wave energy cutoff of $600 \mathrm{eV}$ was used with a $4 \times 4 \times 1$ MonkhorstPack k-point grid ${ }^{35}$ on the (111) facet. The geometry of each system was optimized using a maximum force convergence threshold of $0.02 \mathrm{eV} / \AA$, and a dipole correction was applied in the direction perpendicular to the pyrochlore surface. Surface calculations were performed on 4-layer slabs, where the bottom two layers were frozen to the bulk pyrochlore geometry, and the upper two layers were relaxed. The adsorption energies of each OER intermediate $\left(\Delta \mathrm{G}_{\mathrm{OH}}, \Delta \mathrm{G}_{\mathrm{O}}, \Delta \mathrm{G}_{\mathrm{OOH}}\right)$ were computed by subtracting the DFT-computed 
energies of bare pyrochlore surfaces and gas-phase $\mathrm{H}_{2} \mathrm{O}$ and $\mathrm{H}_{2}$ reference molecules from those of pyrochlore surfaces with each adsorbed intermediate. Energies of all the calculated OER steps are summarized in Table S18 with optimized structures available on the Catalysishub.org repository ${ }^{37}$ under https://www.catalysis-hub.org/publications/HubertAcidic2020. Free energy corrections for each OER adsorbate were computed in the harmonic limit from their respective vibrational modes. Additional details are provided in Table S8.

To evaluate the average $\mathrm{O} 2 p$-state energy for the leached and pristine pyrochlore structures, the first moment of the projected $2 p$ density of states was computed for each adsorbed oxygen according to Eqn. 1, as described in previous reports. ${ }^{38}$ An energy range of $-10 \mathrm{eV}$ to $2 \mathrm{eV}$ with respect to the Fermi level was probed to capture bonding and antibonding $\mathrm{O}$ $2 p$-states. Bader analysis was performed on $\mathrm{Ru}$ active sites via the Bader analysis program for VASP developed by Henkelman et al. ${ }^{39}$

$$
\bar{\epsilon}_{2 p}=\frac{\int_{\epsilon_{\min }}^{\epsilon_{\max }} \rho_{2 p} \epsilon d \epsilon}{\int_{\epsilon_{\min }}^{\epsilon_{\max }} \rho_{2 p} d \epsilon}
$$

\section{Results and Discussion}

\section{Bulk crystalline nanoparticulate $\mathrm{A}_{2} \mathrm{Ru}_{2} \mathrm{O}_{7}$ catalysts synthesized}

In this study, we selected A-site elements ( $\mathrm{Y}, \mathrm{Nd}, \mathrm{Gd}, \mathrm{Bi}$ ) known to form $\mathrm{A}_{2} \mathrm{Ru}_{2} \mathrm{O}_{7}$ phases with varying physical and electronic properties such as A-site ionic radius and bulk conductivity, allowing us to investigate both structural and electronic impacts on OER activity and stability (Table S1). ${ }^{20,40-45}$ We synthesized the pyrochlores using a sol-gel method in which A-site and $\mathrm{Ru}$ nitrate precursors were first mixed in an aqueous environment and then calcined in air at high temperatures $\left(700-1000^{\circ} \mathrm{C}\right)$. The nanoparticulate morphology of the as-synthesized catalysts was observed by scanning electron microscopy (SEM) (Figure 1A-B). Using the Brunauer-Emmett-Teller (BET) method, we found the surface area to be approximately constant $\left(\sim 2-4 \mathrm{~m}^{2} / \mathrm{g}\right)$ across all synthesized materials (Table S2). X-ray 
diffraction (XRD) of the as-synthesized powder catalysts revealed the bulk of each catalyst formed the crystalline pyrochlore phase (Figure 1C). A shift in the XRD patterns to higher $2 \theta$ values with A-site was observed, indicating a decrease in the lattice constant of the pyrochlores, where $\mathrm{Nd}>\mathrm{Bi}>\mathrm{Gd}>\mathrm{Y}$, consistent with other reports ${ }^{42}$ and our DFT-predicted structures (Figure 1D, S1).

Ex-situ x-ray absorption spectroscopy (XAS) measurements were also used to characterize the bulk composition of the powder catalysts. Previous work has shown that meaningful insight regarding OER activity can be drawn from ex-situ O K-edge measurements of asprepared materials, even though the surface under reaction conditions may differ. ${ }^{21,46}$ The two pre-edge features in the $\mathrm{O}$ K-edge spectra at $\sim 529 \mathrm{eV}$ and $532 \mathrm{eV}$ represent the transition of a core $\mathrm{O} 1 s$ electron to $\mathrm{Ru} 4 d t_{2 g}$ and $e_{g}$ states that hybridize with $\mathrm{O} 2 p$ states, respectively ${ }^{22,47,48}$ (Figure 1E). These pre-edge features have previously been used to estimate the extent of hybridization between the $\mathrm{O} 2 p$ and $\mathrm{Ru} 4 d$ orbitals. ${ }^{22,49,50}$ We find that the pre-edge intensities (and areas) change with composition, where $\mathrm{Y}<\mathrm{Nd}<\mathrm{Gd}<\mathrm{Bi}<$ $\mathrm{RuO}_{2}$, indicating that the $\mathrm{Ru} 4 d$ and $\mathrm{O} 2 p$ orbitals have the strongest interaction in $\mathrm{RuO}_{2}$. Interestingly, the shape of the $\mathrm{Bi}_{2} \mathrm{Ru}_{2} \mathrm{O}_{7} \mathrm{O}$ K-edge XAS spectrum more closely resembles that of $\mathrm{RuO}_{2}$ than the other pyrochlore compositions, which suggests the hybridization of its $\mathrm{Ru} 4 d$ and $\mathrm{O} 2 p$ states is more similar to $\mathrm{RuO}_{2}$ than to the other pyrochlores.

Additional characterization of the $\mathrm{Ru}$ K-edge extended x-ray absorption fine structure (EXAFS) region shows that all pyrochlores have 6-fold Ru-O coordination $(\sim 1.53 \AA)$ similar to $\mathrm{RuO}_{2}$ (Figure $\left.1 \mathrm{~F}\right)^{16,51}$ (for k-space plot, refer to Figure S2). The $\mathrm{RuO}_{2}$ features near 2.6 and $3.2 \AA$ represent 2-fold and 8-fold Ru-Ru coordination, respectively, while the single feature at $3.2 \AA$ for $\mathrm{A}_{2} \mathrm{Ru}_{2} \mathrm{O}_{7}$ catalysts represents both $\mathrm{Ru}-\mathrm{Ru}$ and $\mathrm{A}-\mathrm{Ru}$ coordination. ${ }^{16}$ Note that the radial distances in Figure $1 \mathrm{~F}$ are not phase corrected, and the actual bond lengths were determined by fitting the first shell of the EXAFS data (Figure S3). In addition, EXAFS simulations from crystallographic structures agree well with our experimental results, confirming the phase purity of the pyrochlores (Figure S4). From these simulations, we also 
observed that the peak intensity at $3.2 \AA$ in Figure $1 \mathrm{~F}$ depends on the identity of A-site element rather than disorder in the structures. Most notably, we found that $\mathrm{Bi}_{2} \mathrm{Ru}_{2} \mathrm{O}_{7}$ has a much shorter $\mathrm{Ru}-\mathrm{O}$ bond relative to the other $\mathrm{A}_{2} \mathrm{Ru}_{2} \mathrm{O}_{7}$ and is similar in length to the $\mathrm{Ru}-\mathrm{O}$ bond in $\mathrm{RuO}_{2}$ (Figure 1F, Table S3).
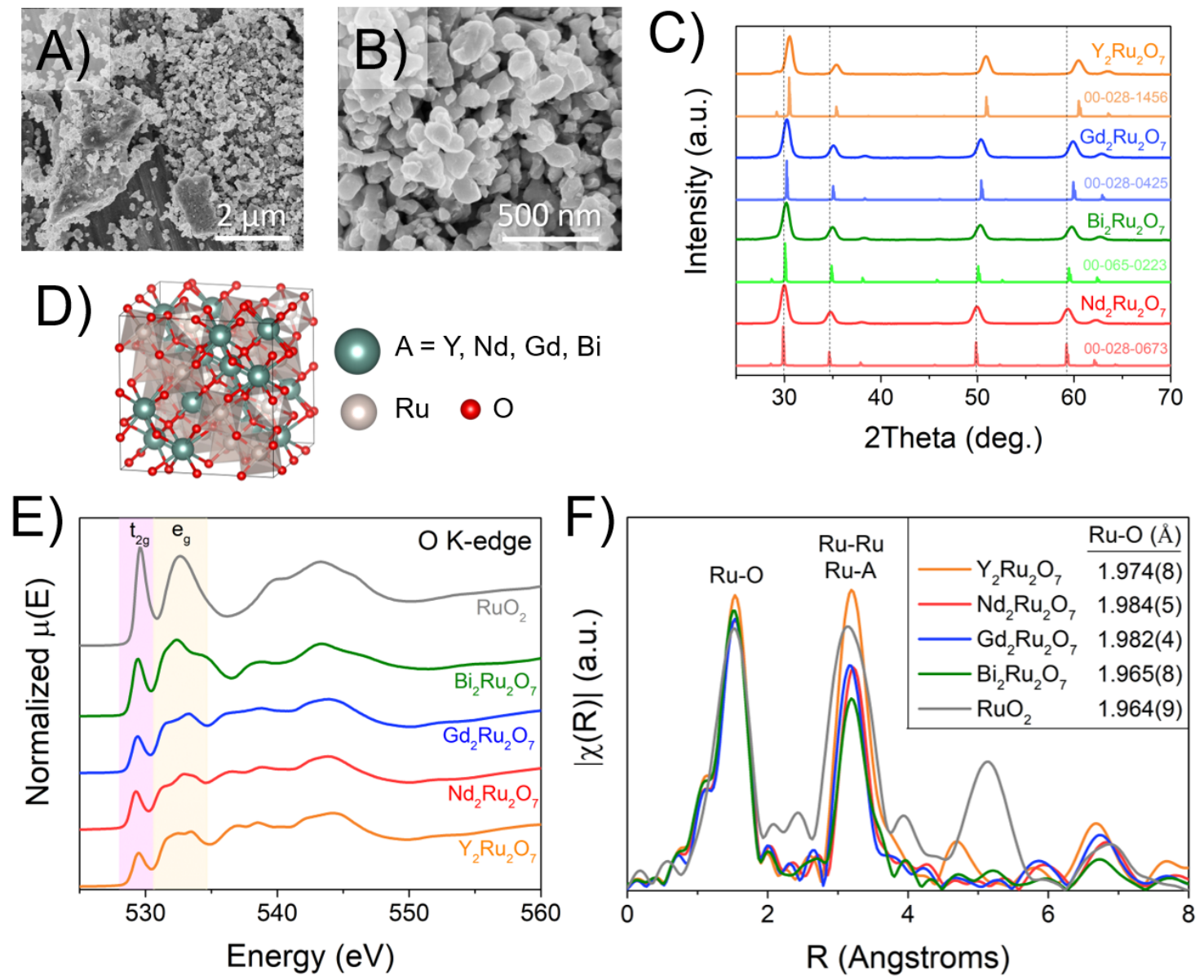

Figure 1: (A-B) Representative SEM images of $\mathrm{Y}_{2} \mathrm{Ru}_{2} \mathrm{O}_{7}$ at two different magnifications. (C) XRD patterns for as-synthesized catalysts (dark) alternating with ICSD reference patterns (light). Dashed lines show $\mathrm{Nd}_{2} \mathrm{Ru}_{2} \mathrm{O}_{7}$ peak positions to highlight the shift with varying Asite. (D) Calculated $\mathrm{A}_{2} \mathrm{Ru}_{2} \mathrm{O}_{7}$ pyrochlore structures exhibit corner-sharing Ru octahedra. (E) Normalized XANES spectra at the O K-edge for all catalyst powders. Purple and yellow shaded regions highlight $\mathrm{Ru} 4 d t_{2 g}$ and $e_{g}$ regions. (F) Fourier transformed EXAFS spectra at the $\mathrm{Ru} \mathrm{K}$-edge for all catalyst powders. Ru-O bond lengths determined by fitting the first shell.

The surface composition of the as-synthesized catalysts was also investigated. X-ray 
photoelectron spectroscopy (XPS) confirmed the molar ratio of A:Ru was $~ 50: 50$ on the surface, consistent with the stoichiometry of the pyrochlore phase (Table S2). The Ru $3 d$ region of $\mathrm{Bi}_{2} \mathrm{Ru}_{2} \mathrm{O}_{7}$ is distinct from the other $\mathrm{A}_{2} \mathrm{Ru}_{2} \mathrm{O}_{7}$ spectra in that it has a second doublet at lower binding energies which has been previously attributed to "screened" Ru $3 d$ orbitals ${ }^{52}$ (Figure S6). This doublet suggests that $\mathrm{Bi}_{2} \mathrm{Ru}_{2} \mathrm{O}_{7}$ is more conductive (similar to $\mathrm{RuO}_{2}$ ), and the absence of this screened doublet in the $\mathrm{Y}, \mathrm{Nd}$, and Gd-containing pyrochlores is indicative of Mott insulators. ${ }^{52}$ This similarity between $\mathrm{Bi}_{2} \mathrm{Ru}_{2} \mathrm{O}_{7}$ and $\mathrm{RuO}_{2}$ is consistent with our O K-edge analysis described above. The appearance of a more reduced Ru oxidation state in $\mathrm{Bi}_{2} \mathrm{Ru}_{2} \mathrm{O}_{7}$ is also reflected in the $\mathrm{Ru} \mathrm{K}$-edge $\mathrm{x}$-ray absorption near edge spectroscopy (XANES) region where it is shifted to a lower pre-edge energy (Figure S5). We note that

although changes in $\mathrm{Ru}$ oxidation state can impact the $\mathrm{O}$ K-edge $\mathrm{Ru} 4 d t_{2 g}$ peak intensity, ${ }^{53}$ we expect this is a much smaller impact compared to $\mathrm{Ru} 4 d-\mathrm{O} 2 p$ orbital hybridization.

\section{$\mathrm{A}_{2} \mathrm{Ru}_{2} \mathrm{O}_{7}$ catalysts have greater specific activity than $\mathrm{RuO}_{2}$}

The OER activity of each $\mathrm{A}_{2} \mathrm{Ru}_{2} \mathrm{O}_{7}$ catalyst was measured in $0.1 \mathrm{M} \mathrm{HClO}_{4}$ using cyclic voltammetry (CV, $10 \mathrm{mV} / \mathrm{s}, 10$ cycles) and compared to a commercial $\mathrm{RuO}_{2}$ powder as a standard (Figure 2). All catalyst inks contained Vulcan carbon to improve the dispersion of the catalyst and enhance the electrical connection between the catalyst and the substrate (Figure S7). The activity of the Vulcan carbon itself was minimal in the voltage window of interest (Figure S8B). At least 3 independent CV experiments were conducted for each catalyst, and representative data from one CV experiment is shown in the supporting information for each pyrochlore (Figure S9). The measured current was normalized by the BET surface area of the powders (Table S2). This normalization method accounts for the different surface area (and presumed number of available active sites) across catalyst samples and is used as a measure of intrinsic catalyst activity for nanoparticulate catalysts. ${ }^{16,46}$ Figures $2 \mathrm{~A}$ and $2 \mathrm{~B}$ illustrate the average current and standard deviation for the forward sweep of the 1st and 10th CVs of each catalyst, respectively. For ease of comparison to other literature 
reports, Figure S10 shows this CV data re-normalized by geometric area and mass of Ru and Table S6 tabulates the potential at which each catalyst achieves $10 \mathrm{~mA} / \mathrm{cm}^{2}$ geo.

Similar to other reports, ${ }^{16}$ each $\mathrm{A}_{2} \mathrm{Ru}_{2} \mathrm{O}_{7}$ material showed superior specific activity compared to $\mathrm{RuO}_{2}$ after 1, 3, and $10 \mathrm{CVs}$ (Figure 2C). Additionally, the specific activity appeared to depend on the A-site element in the pyrochlore. In the 1st CV, the trend in specific activity at $1.55 \mathrm{~V}$ vs. RHE was $\mathrm{RuO}_{2}<\mathrm{Bi}<\mathrm{Gd}<\mathrm{Y}<\mathrm{Nd}$ (Figure 2C). After $10 \mathrm{CVs}$, the specific activity of each catalyst appeared to approach a steady-state value, resulting in a slightly different trend $\left(\mathrm{RuO}_{2}<\mathrm{Bi}<\mathrm{Gd}<\mathrm{Nd}<\mathrm{Y}\right)$ as $\mathrm{Nd}_{2} \mathrm{Ru}_{2} \mathrm{O}_{7}$ activity decreased. This same trend with A-site element is also reflected in the Tafel slopes of the 10th CV, albeit across a relatively narrow range of values $(40-48 \mathrm{mV} /$ decade). The similarity in Tafel slope values may indicates that a similar OER reaction mechanism occurs on each $\mathrm{A}_{2} \mathrm{Ru}_{2} \mathrm{O}_{7}$ after 10 CVs (Figure S11).

We highlight that $\mathrm{Y}, \mathrm{Nd}$, and Gd-containing pyrochlores exhibit greater activity than $\mathrm{Bi}_{2} \mathrm{Ru}_{2} \mathrm{O}_{7}$ and $\mathrm{RuO}_{2}$ during all CVs. We find that this difference in initial OER activity correlates well with the ex-situ XAS measurements at the Ru and O K-edges (Figure 1E, 1F). Our characterization of the as-prepared catalysts shows that a short Ru-O bond and strong interaction of the $\mathrm{Ru} 4 d$-O $2 p$ orbitals, such as in $\mathrm{RuO}_{2}$ and $\mathrm{Bi}_{2} \mathrm{Ru}_{2} \mathrm{O}_{7}$, results in lower OER activity relative to the other $\mathrm{A}_{2} \mathrm{Ru}_{2} \mathrm{O}_{7}$ catalysts (Figure 2D). This insight is consistent with reports of similar Ru-based OER catalysts, where enhanced activity is attributed to shifting the $\mathrm{Ru} 4 d$ band center to lower values. ${ }^{22,38}$ While A-site ionic radius and lattice parameters have previously rationalized trends in OER activity, ${ }^{11,12}$ the A-site can lead to additional structural effects, such as distortion and varying relative orientation of the $\mathrm{RuO}_{6}$ octahedra within the pyrochlores. ${ }^{44,54,55}$ This indicates that A-site ionic radius and lattice constant do not always correlate with Ru-O bond length. ${ }^{42}$ Thus, we find that Ru-O bond length to be a better indicator of initial OER activity for the $\mathrm{A}_{2} \mathrm{Ru}_{2} \mathrm{O}_{7}$ catalysts studied herein. We also acknowledge and emphasize that catalytic activity trends may change over time, which could explain different observations reported in literature. Collectively, our results indicate that 
the composition of $\mathrm{A}_{2} \mathrm{Ru}_{2} \mathrm{O}_{7}$ catalysts impacts both structural and electronic properties, which have a combined influence on the active $\mathrm{Ru}$ sites.
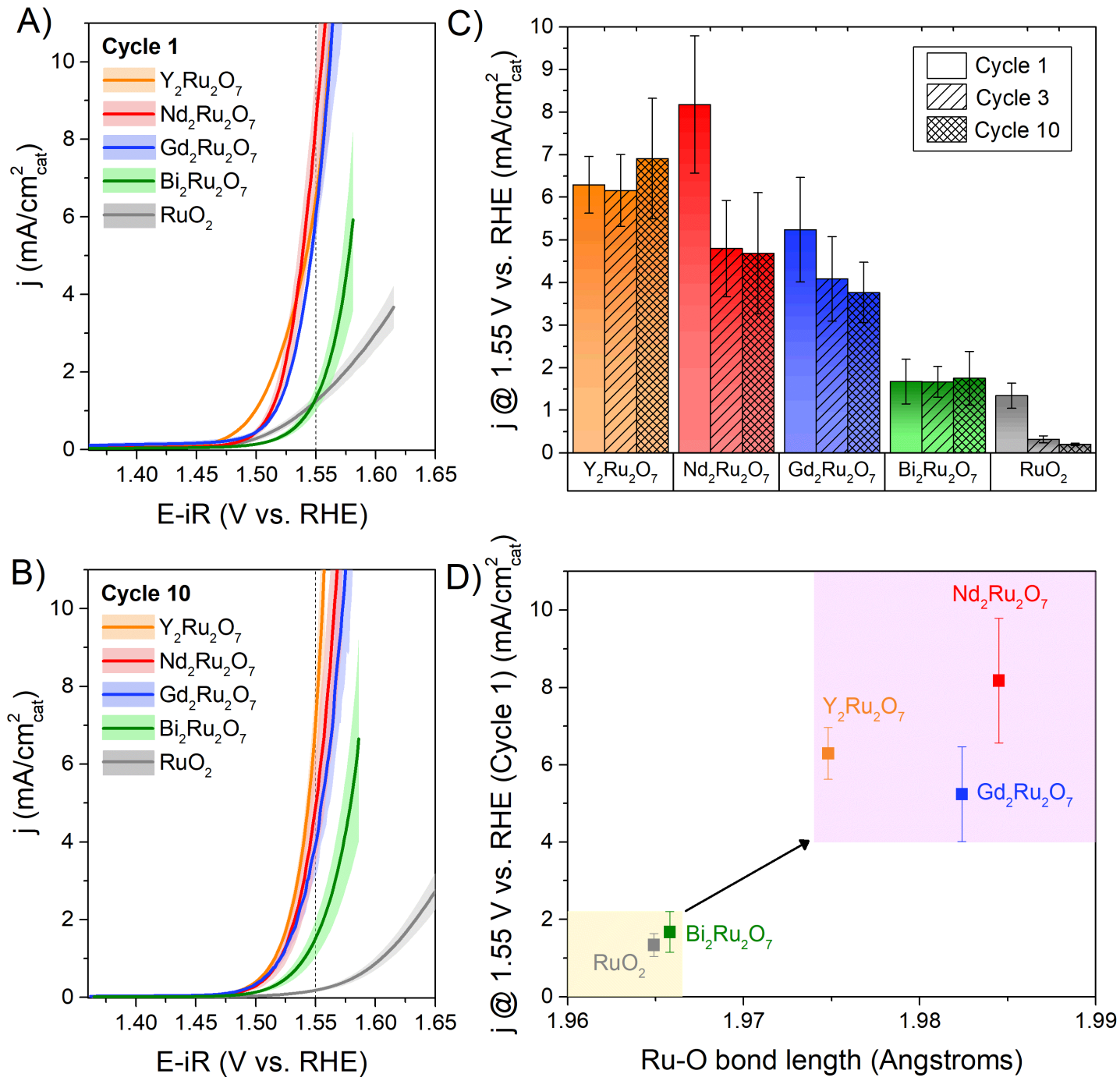

Figure 2: Forward sweep of 1st (A) and 10th (B) CV for each catalyst. Current normalized by BET surface area of powder catalysts. Solid lines represent average values and shaded regions represent standard deviation above/below the average determined from at least 3 independent measurements. Dashed line at $1.55 \mathrm{~V}$ vs. RHE for reference. (C) Bars represent average specific activity after 1, 3, and $10 \mathrm{CVs}$ at $1.55 \mathrm{~V}$ vs. RHE and error bars represent standard deviation from the average determined from at least 3 independent measurements. All CVs collected at $10 \mathrm{mV} / \mathrm{s}$ scan rate in $0.1 \mathrm{M} \mathrm{HClO}_{4}$. (D) Current normalized by BET surface area for the 1 st $\mathrm{CV}$ at $1.55 \mathrm{~V}$ vs. RHE as a function of Ru-O bond length determined from $\mathrm{Ru}$ K-edge EXAFS fits.

The change in activity with subsequent CVs highlights the influence of catalyst stability 
on the apparent catalytic activity. The intrinsic catalyst activity, number of active sites, oxidation of the catalyst support, or catalyst dissolution may all contribute in some part to the measured current. (For additional discussion, please refer to the "Undesired Electrochemical Processes" section in the supporting information.) For example, it is well-known that the initially high current of $\mathrm{Ru}$ metal is due to $\mathrm{Ru}$ oxidation and subsequent dissolution. ${ }^{56-58}$ Here, $\mathrm{Nd}_{2} \mathrm{Ru}_{2} \mathrm{O}_{7}$ and $\mathrm{Gd}_{2} \mathrm{Ru}_{2} \mathrm{O}_{7}$ show some loss in current density after the 1 st $\mathrm{CV}$ before reaching more stable performance after 3 cycles (Figure $2 \mathrm{C}$ ). We speculate that some of the initial current may be due to catalyst dissolution and subsequent surface reconstruction rather than OER. ${ }^{12}$ To investigate this hypothesis, we used chronoamperometry coupled with ICP-MS analysis of the electrolyte to quantify catalyst dissolution over time.

\section{All $\mathrm{A}_{2} \mathrm{Ru}_{2} \mathrm{O}_{7}$ catalysts lose A-site and $\mathrm{Ru}$ atoms during OER}

Catalyst stability was assessed using chronoamperometry (CA) experiments with a constant electrochemical potential of $1.56 \mathrm{~V}$ vs. RHE for a duration of $10 \mathrm{hr}$ in $0.1 \mathrm{M} \mathrm{HClO}_{4}$. By holding an applied potential, all catalysts were subjected to the same oxidizing environment. We note that this method is distinct and complementary to the insight gained from CV cycling. CV cycling repeatedly exposes the catalyst to less oxidizing potentials, simulating intermittent device operation, while a CA simulates continuous operation. Due to the difference in protocols, trends in catalyst stability may differ.

Similar to the time-dependent activity observed during CV testing (Figure 2C), the geometric current density of each catalyst was dynamic with time during the CA (Figure 3A). The geometric current density of each pyrochlore increased ( $10-100 \%)$ within the first hr of testing (Figure S12), but ultimately decreased to 4-51\% of the peak current density after $10 \mathrm{hr}$. Most notably, the current density of $\mathrm{Nd}_{2} \mathrm{Ru}_{2} \mathrm{O}_{7}$ increased by $8 \mathrm{~mA} / \mathrm{cm}^{2}$ geo in the first

$40 \mathrm{~min}$, then decreased at an average rate of $3 \mathrm{~mA} / \mathrm{cm}^{2}$ geo $/ \mathrm{hr}$ over $5 \mathrm{hr}$, finally approaching the current density of $\mathrm{RuO}_{2}$ after $10 \mathrm{hr}$. This dynamic behavior of the $\mathrm{Nd}_{2} \mathrm{Ru}_{2} \mathrm{O}_{7}$ catalyst during OER has been previously attributed to catalyst dissolution followed by surface 
reconstruction ${ }^{12}$ and/or surface roughening. ${ }^{59}$
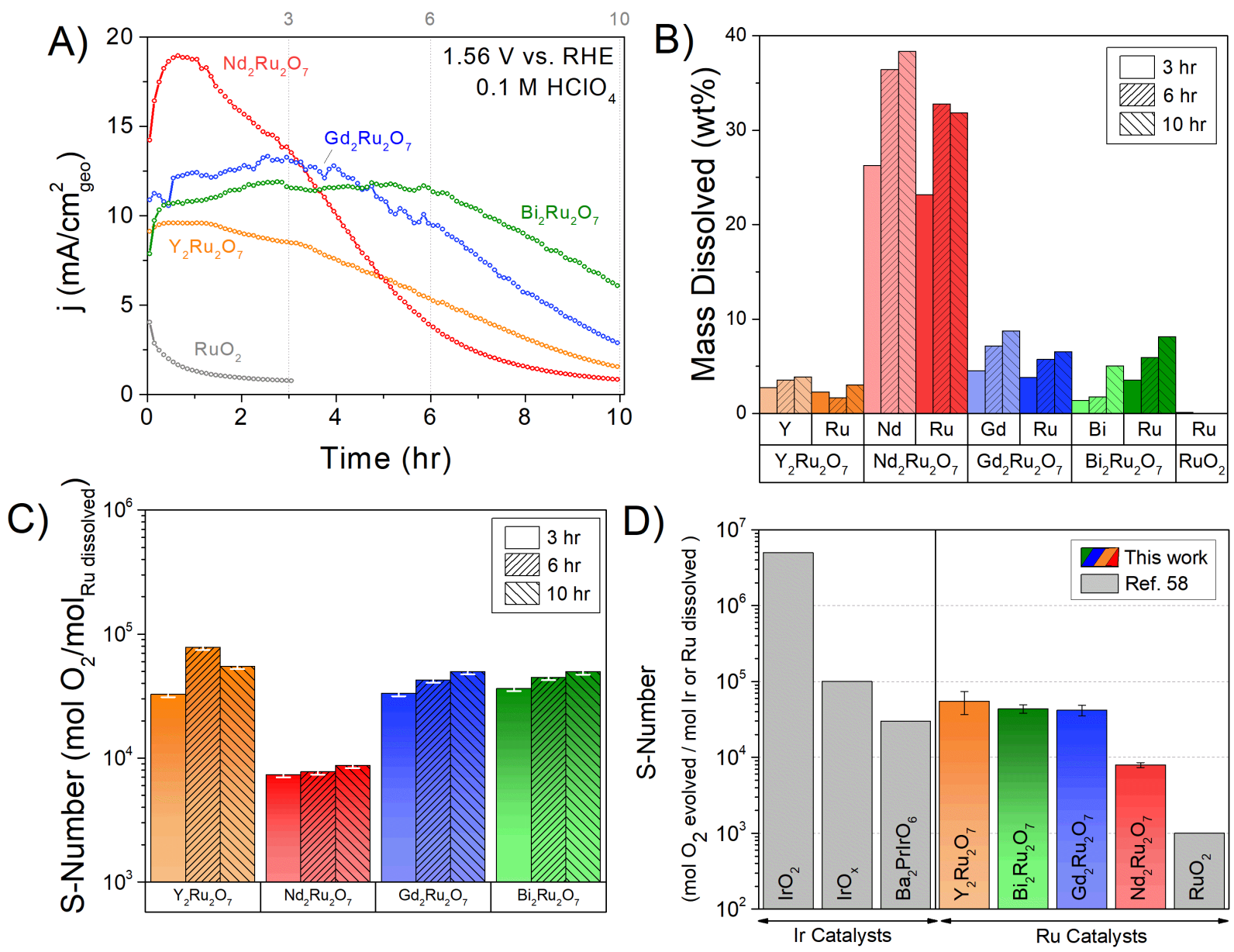

Figure 3: (A) Chronoamperometry measurement of all catalysts at $1.56 \mathrm{~V}$ vs. RHE in 0.1 $\mathrm{M} \mathrm{HClO}_{4}$. Current normalized by geometric area shows dynamic behavior over time. (B) Mass fraction of total deposited catalyst (A-site and $\mathrm{Ru}$ ) that dissolved in the electrolyte after 3, 6, and $10 \mathrm{hr}$. (C) Stability number (S-number) calculated from measured current and total Ru mass dissolved after 3, 6, and $10 \mathrm{hr}$. Error bars (white) indicate S-number for $95 \%$ Faradaic efficiency. (D) Colored bars (this work) represent average S-number from 3, 6, and $10 \mathrm{hr}$ measurements with error bars showing the standard deviation. Gray bars represent values from Ref. 60 .

There are many factors that can impact the measured current density over time, including delamination, agglomeration, or dissolution of the catalyst, as well as substrate or support corrosion. These processes can impact both the number of available catalytic sites and the intrinsic activity of those sites. In this work, we quantify stability by measuring catalyst 
dissolution and qualitatively look for signs of the other degradation mechanisms. We detected negligible dissolution using ICP-MS when all powder catalysts were submerged in $0.1 \mathrm{M}$ $\mathrm{HClO}_{4}$ for $12 \mathrm{hr}$ under no applied potential (Table S7). This demonstrates the high chemical stability of pyrochlores in acidic electrolyte.

We further assessed the catalysts' electrochemical stability during OER catalysis to better understand the relationship between OER activity and stability. We quantified catalyst dissolution during the CA test by performing ICP-MS analysis on electrolyte aliquots taken after 3, 6, and $10 \mathrm{hr}$. As a result of the catalysts' high chemical stability, we assumed that any material that may have mechanically detached from the electrode would not significantly dissolve, and therefore would not be detected in our ICP-MS analysis. Dissolution of both the A-site and $\mathrm{Ru}$ was observed for all catalysts, where the A-site dissolved to a greater extent than $\mathrm{Ru}$ ( $\sim 1.2$ times on average) for $\mathrm{Y}, \mathrm{Nd}$, and Gd-containing pyrochlores (Figure 3B). This preferential A-site dissolution suggests the formation of a Ru-enriched surface during catalysis. Contrary to this trend, more $\mathrm{Ru}$ dissolved than $\mathrm{Bi}$ from $\mathrm{Bi}_{2} \mathrm{Ru}_{2} \mathrm{O}_{7}$, which could indicate the formation of a different surface composition during catalysis. We note that the amount of $\mathrm{Ru}$ dissolved from $\mathrm{RuO}_{2}$ was at the limit for reliable quantification, and that such a small amount of $\mathrm{Ru}$ dissolution from standard $\mathrm{RuO}_{2}$ nanoparticles is expected based on previous reports. ${ }^{56,58,59}$

The rate of catalyst dissolution decreased over time, coincident with a decrease in the measured current density (Figure 3A, Figure S13). Qualitatively, this result suggests that dissolution occurs predominantly when the catalyst is evolving oxygen. ${ }^{60,61}$ To further illustrate this point, we calculated the stability number (S-number), a metric defined as the moles of $\mathrm{O}_{2}$ evolved $\left(\mathrm{n}_{\mathrm{O}_{2}}\right)$ normalized by the moles of $\mathrm{Ru}$ dissolved $\left(\mathrm{n}_{\mathrm{Ru}}\right)$, after 3,6 , and $10 \mathrm{hr}$ of testing (cumulative). ${ }^{60}$ We assume 95-100\% Faradaic efficiency to OER based on a control CA experiment with only Vulcan carbon (no catalyst) on a Au substrate (Figure S8A) and an estimation of the maximum amount of charge required to dissolve all of the catalyst. For further discussion, please refer to the supporting information. 


$$
\text { S-number }=n_{O_{2}} / n_{R u}
$$

Using the S-number metric, we can quantitatively compare catalyst activity $\left(\mathrm{O}_{2}\right.$ evolution rate) to stability ( $\mathrm{Ru}$ dissolution rate), essentially combining the results from Figures 3A and 3B. Interestingly, the calculated S-number for all pyrochlores remained relatively constant with time (Figure 3C), supporting our claim that Ru dissolves predominantly when the catalysts facilitate the OER. Additionally, we see that despite differences in the peak current densities achieved $(\mathrm{Nd}>\mathrm{Gd}>\mathrm{Bi}>\mathrm{Y}$, Figure $3 \mathrm{~A})$, when normalized by the amount of Ru dissolved, the pyrochlores containing $\mathrm{Y}, \mathrm{Gd}$, and $\mathrm{Bi}$ all demonstrate comparable stability, with S-numbers $\sim 5 \times 10^{4} \mathrm{~mol}_{\mathrm{O}_{2}} / \mathrm{mol}_{\text {Ru dissolved }}$ (Figure 3C). In other words, despite their differences in activity, they generate similar amounts of $\mathrm{O}_{2}$ for each $\mathrm{Ru}$ atom dissolved.

In contrast, $\mathrm{Nd}_{2} \mathrm{Ru}_{2} \mathrm{O}_{7}$ appears less electrochemically stable $\left(\sim 8 \mathrm{x}^{3} 0^{3} \mathrm{~mol}_{\mathrm{O}_{2}} / \mathrm{mol}_{\text {Ru dissolved }}\right)$ than the other $\mathrm{A}_{2} \mathrm{Ru}_{2} \mathrm{O}_{7}$, generating nearly 10 times less $\mathrm{O}_{2}$ per $\mathrm{Ru}$ atom dissolved. The instability of $\mathrm{Nd}_{2} \mathrm{Ru}_{2} \mathrm{O}_{7}$ has been previously attributed to the large size of the $\mathrm{Nd}$ atom causing strain that weakens the pyrochlore structure. ${ }^{12}$ We also observe that $\mathrm{Nd}_{2} \mathrm{Ru}_{2} \mathrm{O}_{7}$ has the largest lattice spacing (Figure 1A) and $\mathrm{Ru}-\mathrm{O}$ bond length (Figure $1 \mathrm{~F}$ ) of the pyrochlores studied in this work. However, we do not observe an obvious trend between lattice spacing or $\mathrm{Ru}-\mathrm{O}$ bond length and electrochemical stability (S-number) across all $\mathrm{A}_{2} \mathrm{Ru}_{2} \mathrm{O}_{7}$ compositions studied herein. We also do not observe a clear trend between the S-number and O $2 p$-Ru $4 d$ orbital hybridization (Figure 1B). Our results suggest that stability is controlled by a combination of structural and electronic parameters.

Most notably, all $\mathrm{A}_{2} \mathrm{Ru}_{2} \mathrm{O}_{7}$ catalysts investigated demonstrated greater stability (and activity) than $\mathrm{RuO}_{2}$ (Figure 3D). In the context of other OER catalysts, the S-numbers of the Ru-based pyrochlore catalysts in this work even approach that of Ir-based mixed metal oxides $\left(10^{4}-10^{5}\right)$. While not achieving the stability of $\mathrm{IrO}_{2}$, the stability of these $\mathrm{A}_{2} \mathrm{Ru}_{2} \mathrm{O}_{7}$ catalysts suggests that changing the composition of $\mathrm{Ru}$ oxide materials is a promising strategy to stabilize active Ru sites for OER in acidic conditions. Our results also indicate that 
electrochemical stability and rate of catalyst dissolution cannot be determined from individual structural or electronic properties alone. We further examine the thermodynamics of catalyst dissolution computationally through Pourbaix analysis below. However, we will first discuss experimental methods used to probe the catalyst structure during and after electrochemical testing to gain insight about intrinsic activity and stability.

\section{Catalyst Structure During and After Testing}

To further probe catalyst degradation mechanisms, we investigated the structure and composition of the catalyst after CA testing. By XRD, we confirm that all post-test catalysts (on a $\mathrm{Au}$ disk substrate after $10 \mathrm{hr} \mathrm{CA}$ ) retain the bulk crystalline pyrochlore phase (Figure 4A, Figure S14). This result, in combination with the ICP-MS measurements, indicates that dissolution occurs from the surface of the catalyst nanoparticles. Consistent with XRD measurements, STEM-EDS composite images of $\mathrm{Y}_{2} \mathrm{Ru}_{2} \mathrm{O}_{7}$ before and after CA testing illustrate the presence and distribution of $\mathrm{Y}, \mathrm{Ru}$, and $\mathrm{O}$ in the nanoparticles (Figure 4B,C). STEM-ADF images of both pre- and post-test samples are shown in Figure S15. HR-TEM analysis of $\mathrm{Nd}_{2} \mathrm{Ru}_{2} \mathrm{O}_{7}$ before and after $\mathrm{CA}$ testing was conducted to investigate changes in surface morphology. While significant changes were not observed, the heterogeneity of the nanoparticles render these results inconclusive (Figure S16-S18).

In an effort to observe changes to the catalyst during OER, we collected in-situ XAS measurements at the $\mathrm{Ru} \mathrm{K}$-edge for $\mathrm{Y}_{2} \mathrm{Ru}_{2} \mathrm{O}_{7}$. Details of the experimental setup can be found in the supporting information. In summary, a series of potentials were held (open circuit voltage, $1.27 \mathrm{~V}, 1.47 \mathrm{~V}, \sim 1.6 \mathrm{~V}, 1.18 \mathrm{~V}$ vs. RHE, and open circuit voltage again) while XAS measurements were collected (Figure S19B). At the end of the experiment, 3\% of the $\mathrm{Y}$ and $\sim 2 \%$ of the $\mathrm{Ru}$ from the catalyst had dissolved. We did not observe significant changes in the Ru K-edge EXAFS spectra with changing voltage or time despite our detection of some catalyst dissolution (Figure S19C). The EXAFS signal contains information about the bulk and surface regions, including both crystalline and possible amorphous phases. 
Our finding complements the post-test XRD results (Figure 4A), and we conclude that the bulk $\mathrm{Y}_{2} \mathrm{Ru}_{2} \mathrm{O}_{7}$ material is stable during OER catalysis. With such large nanoparticles, the XAS signal from the surface would only constitute a small fraction of the overall signal, and therefore, any changes at the immediate surface were likely not measurably detected by this technique.

Although changes to catalyst surface morphology were not directly observed via ex-situ TEM or in-situ XAS, both of these methods and XRD indicate that the bulk pyrochlore phase remains intact after testing. Combined with the observed non-stoichiometric dissolution of the catalysts via ICP-MS, we infer that their surfaces evolved during OER. This is consistent with reports of other mixed metal oxide materials under OER conditions. ${ }^{10,13}$
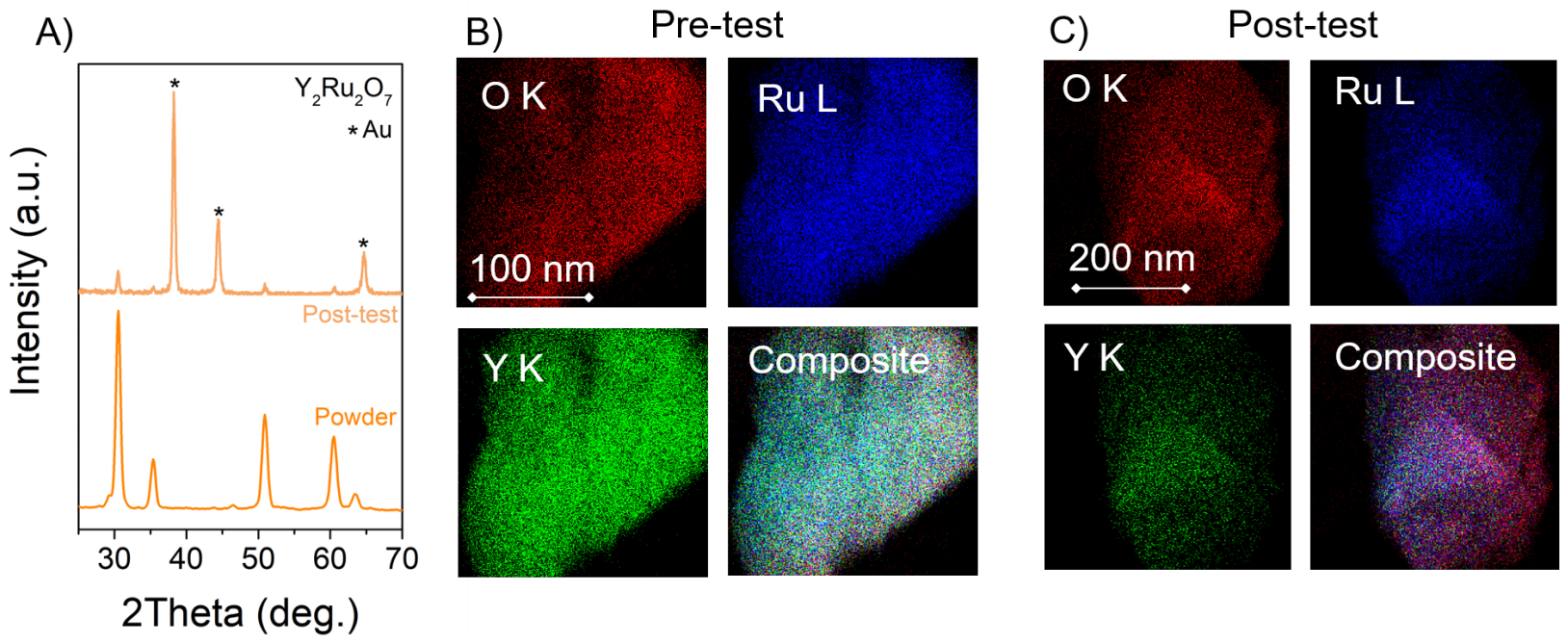

Figure 4: (A) Representative XRD for $\mathrm{Y}_{2} \mathrm{Ru}_{2} \mathrm{O}_{7}$ deposited on Au substrate after CA testing compared to the as-synthesized powder shows that bulk pyrochlore structure remains. XRD peaks marked ${ }^{*}$ correspond to Au substrate. STEM-EDS mappings of $\mathrm{Y}_{2} \mathrm{Ru}_{2} \mathrm{O}_{7}$ before (B) and after (C) CA testing. Composite images show the overlap of $\mathrm{Y}$ (green), Ru (blue), and $\mathrm{O}$ (red).

We additionally estimated the OER turnover frequency (TOF, $\mathrm{O}_{2} / \mathrm{s} / \mathrm{Ru}$ ) after $0,3,6$, and $10 \mathrm{hr}$ of CA testing, adjusting the number of active Ru atoms to account for dissolution (Figure S20). The decrease in TOF after $3 \mathrm{hr}$ suggests that degradation mechanisms beyond simply the loss of $\mathrm{Ru}$ active sites, such as a decrease in available surface area, contributed to the observed decrease in measured current. We further note that the increase in TOF in 
the first $3 \mathrm{hr}$ could be explained by enhanced intrinsic catalytic activity or an increase in electrochemically active surface area. Details of the TOF calculation can be found in the supporting information.

To investigate how support corrosion and catalyst delamination may have impacted the change in current density during the CA experiment, we used scanning electron microscopy (SEM) and Auger electron spectroscopy (AES). Prior to electrochemical CA testing, SEM shows the catalyst embedded within the Vulcan carbon support, while SEM images and AES maps after testing show much less Vulcan carbon on the Au substrate (Figure S21-S22). We speculate that the oxidation or delamination of the carbon support could lead to a reduced or limited conductive pathway between the catalyst and the Au substrate, resulting in a reduction in the number of catalytically active sites and hence contributing to the observed decrease in current density over $10 \mathrm{hr}$.

Overall, we have observed experimentally that the A-site element in $\mathrm{A}_{2} \mathrm{Ru}_{2} \mathrm{O}_{7}$ can influence OER activity and stability. Specifically, under the same applied electrochemical potential, $\mathrm{Nd}_{2} \mathrm{Ru}_{2} \mathrm{O}_{7}$ dissolves $\sim 10$ times faster per mole of $\mathrm{O}_{2}$ evolved than the $\mathrm{Y}_{2} \mathrm{Ru}_{2} \mathrm{O}_{7}$, $\mathrm{Gd}_{2} \mathrm{Ru}_{2} \mathrm{O}_{7}$, and $\mathrm{Bi}_{2} \mathrm{Ru}_{2} \mathrm{O}_{7}$ pyrochlores studied in this work. To understand if this difference in kinetics was driven purely by differences in the thermodynamic driving forces for dissolution, we theoretically modeled the $\mathrm{A}_{2} \mathrm{Ru}_{2} \mathrm{O}_{7}$ catalysts to predict the most stable structures under acidic OER conditions.

\section{Pourbaix Analysis reveals Thermodynamic Driving Force for Py- rochlore Degradation}

The thermodynamic stability of the structures considered in this study was assessed via Pourbaix analysis, where the free energies of the pyrochlore phases were calculated at varying

$\mathrm{pH}$ and electrochemical potentials in aqueous conditions. ${ }^{36,62-64}$ Pourbaix diagrams were constructed from the DFT-predicted formation energies of both the pyrochlore structures and additional solvated and solid species containing elements that comprise each pyrochlore 
phase (Figure 5). These additional structures were obtained from the Materials Project ${ }^{65}$ and Inorganic Crystal Structure Databases (ICSD) ${ }^{66}$ and optimized according to the DFT methods described in the "Formation Energy Computation for Pourbaix Analysis" section of the supporting information. In addition to the species within these databases, several Ru-based pyrochlore-like structures with leached A-site cations and hydroxylated O were considered, as depicted in Figure S23. The domains within the Pourbaix diagrams represent the most stable combination of species at each potential and $\mathrm{pH}$ condition.

As an extension of the typical Pourbaix analysis, we also considered the possibility of metastable phases resulting from large kinetic barriers of structural reorganization. Previous reports suggest that metastable phases may exist with free energies up to $0.5 \mathrm{eV} /$ atom greater than the most stable species at a given reaction condition. ${ }^{67}$ To address this possibility, we determined the free energy difference between the pyrochlore structure and the most energetically favorable species at each point in Pourbaix space $\left(\Delta \mathrm{G}_{\mathrm{pbx}}\right),{ }^{67}$ as represented by the colormaps overlaid on the Pourbaix diagrams (Figure 5).

At OER-relevant potentials ( $\sim 1.56 \mathrm{~V}$ vs. RHE) in acidic conditions, the most stable states of the A-site species in the pyrochlores are aqueous ions, suggesting that these species are prone to leaching. In accordance with experimental reports, the most stable $\mathrm{Ru}-$ containing species in each case is $\mathrm{RuO}_{4} \cdot{ }^{68-70}$ As indicated by the red regions in Figure 5, it is clear from our Pourbaix analysis that all of the $\mathrm{A}_{2} \mathrm{Ru}_{2} \mathrm{O}_{7}$ structures considered in this study exceed the $+0.5 \mathrm{eV} /$ atom empirical metastability threshold from previous reports ${ }^{67}$ under acidic OER conditions. The thermodynamic analysis thus indicates that all materials in this study are ultimately expected to dissolve during the OER, as is the case with pure $\mathrm{RuO}_{2}$. For instance, within the Bi-containing system, the most stable combination of species at $1.56 \mathrm{~V}$ vs. $\mathrm{RHE}$ and $\mathrm{pH} 1$ is $\mathrm{RuO}_{4}$ and $\mathrm{BiO}^{+}$(Figure 5B). However, in this particular case, solid $\mathrm{BiO}_{2}$ and $\mathrm{Bi}_{4} \mathrm{O}_{7}$ from neighboring Pourbaix domains exhibit $\Delta \mathrm{G}_{\mathrm{pbx}}$ values less than $0.1 \mathrm{eV}$, indicating that a phase transformation from $\mathrm{Bi}_{2} \mathrm{Ru}_{2} \mathrm{O}_{7}$ to these species may lead to metastability under these conditions. These potentially metastable Bi oxide phases and the 
relatively lower thermodynamic driving force for decomposition of $\mathrm{Bi}_{2} \mathrm{Ru}_{2} \mathrm{O}_{7}(0.6 \mathrm{eV} /$ atom) compared to the other pyrochlores provide possible explanations for the lower experimental leaching rate of Bi relative to $\mathrm{Ru}$ (Figure 3B).

Overall, our analysis reveals a significant thermodynamic driving force for both A-site cation and $\mathrm{Ru}$ leaching, which corroborates experimental evidence of catalyst dissolution under acidic oxidizing conditions (Figure 3B). To compare to our experimental results, we determined the trend in thermodynamic driving force for dissolution of each A-site at $1.56 \mathrm{~V}$ vs. RHE and pH 1 to be $\mathrm{Bi}<\mathrm{Gd} \sim \mathrm{Y} \sim \mathrm{Nd}$, as quantified in Table S11. The considerable decomposition driving force of $1.3 \mathrm{eV} /$ atom for $\mathrm{Nd}_{2} \mathrm{Ru}_{2} \mathrm{O}_{7}$ aligns with the high experimentally measured dissolution rates for this material. However, this thermodynamic trend does not fully explain the trends in stability observed experimentally, in particular the similar S-number of Y, Gd, and Bi-containing pyrochlores (Figure 3C). Thus, a kinetic analysis of dissolution mechanisms would be required for a more complete theoretical description of the relative dissolution rates of the pyrochlore structures. Additional future efforts could also involve understanding the dynamics of structure and composition of the catalyst surface during reaction.

As suggested by previous reports, the relative leaching rates of A-site cations versus $\mathrm{Ru}$ atoms from the pyrochlore structure can lead to the formation of amorphous hydrous overlayers. ${ }^{10,71}$ Our experimental results also suggest that the identity of the A-site element affects the rate of dissolution, and therefore the formation rate of such phases. We predict that this dissolution process during OER exposes highly oxidized Ru active sites, which we investigate below. 
A)

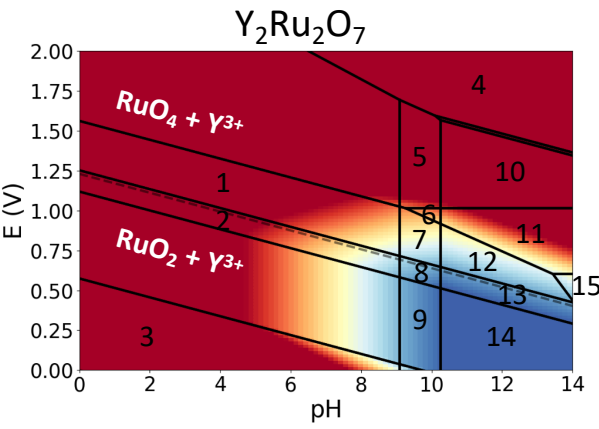

C)

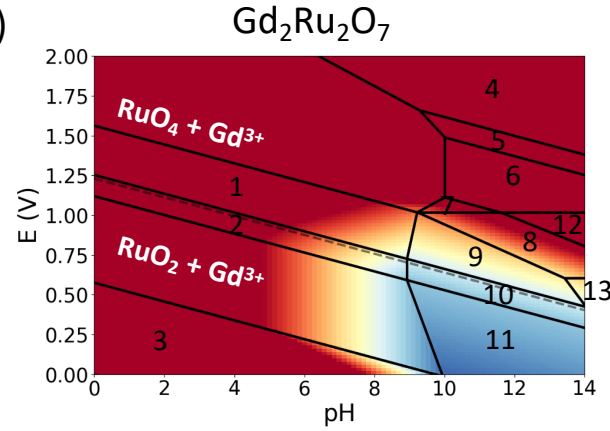

B)

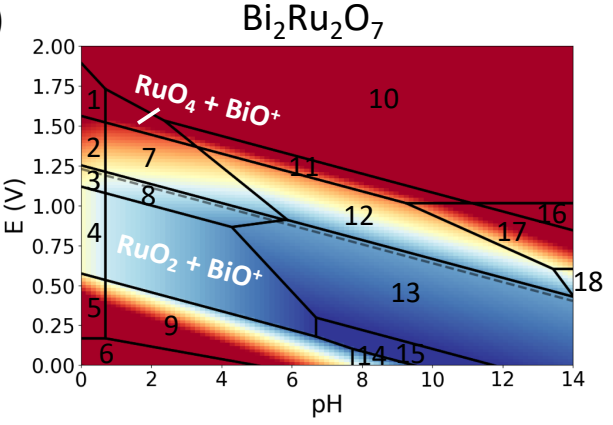

D)

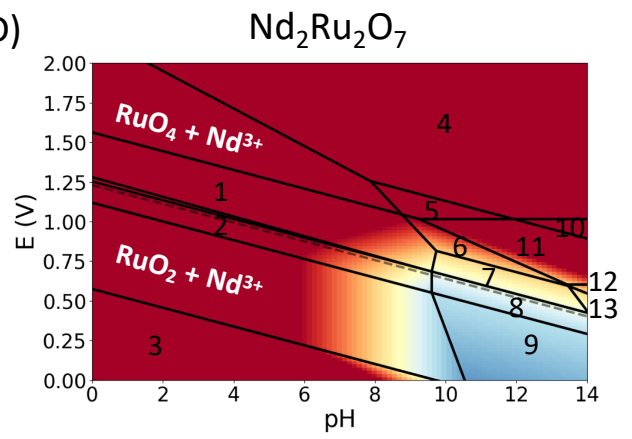

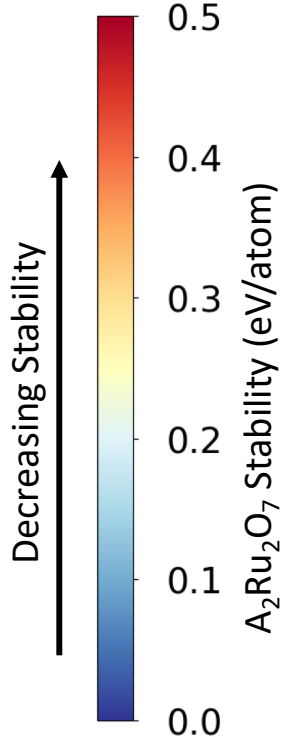

0.0

Figure 5: Calculated Pourbaix diagrams of (A) Y-Ru ; (B) Bi-Ru; (C) Gd-Ru; (D) Nd-Ru systems. Ion concentrations are $10^{-6} \mathrm{M}$, and element compositions are set to that of the pyrochlore phase. The numbered stability domains are labeled in Tables S12 - S15, and the free energies used to construct this plot are tabulated in Table S10.

\section{A-site Cation Leaching Predicted to Expose Active OER Sites}

The experimentally observed dissolution of the pyrochlores and initial rise in current density (and TOF) during CA measurements indicate that these catalyst surfaces undergo dynamic transformations that are linked to their OER activity. While precise theoretical models of the transient pyrochlore surfaces would necessitate detailed in-situ characterization, theoretical predictions of OER activity trends for given active sites upon dissolution can complement our understanding of the dynamic catalyst surface. A complete theoretical understanding of the OER activity of these systems would require a microkinetic model incorporating activation barriers of reaction steps. However, previous reports have demonstrated that activity predictions from a thermodynamic description of elementary reaction steps align well with those from microkinetic modeling, ${ }^{72}$ meaning that significant insight may be gained from thermodynamic analysis. ${ }^{73}$ Therefore, we predicted the activity of the pyrochlores studied 
herein using DFT to evaluate the thermodynamic reaction energetics of the 4-electron OER mechanism ${ }^{74}$ described by Eqns. $3-6$ and illustrated in Figure 6A. By employing the computational hydrogen electrode ${ }^{74}$ to describe the potential dependence of each reaction step, we define the theoretical OER overpotential as the minimum potential at which all of the reaction steps are thermodynamically favorable $(\Delta \mathrm{G} \leq 0 \mathrm{eV})$.

$$
\begin{gathered}
\mathrm{H}_{2} \mathrm{O} \rightleftharpoons{ }^{*} \mathrm{OH}+\mathrm{H}^{+}+e^{-} \\
{ }^{*} \mathrm{OH} \rightleftharpoons{ }^{*} \mathrm{O}+\mathrm{H}^{+}+e^{-} \\
{ }^{*} \mathrm{O}+\mathrm{H}_{2} \mathrm{O} \rightleftharpoons{ }^{*} \mathrm{OOH}+\mathrm{H}^{+}+e^{-} \\
{ }^{*} \mathrm{OOH} \rightleftharpoons \mathrm{O}_{2}+{ }^{*}+H^{+}+e^{-}
\end{gathered}
$$

Based on previously reported trends in OER activity for coordinatively unsaturated (CUS) $\mathrm{Ru}$ active sites on $\mathrm{RuO}_{2},{ }^{75-77}$ we computed the OER activity of unsaturated $\mathrm{Ru}$ active sites on the (111) facet of the pyrochlore structures depicted in Figure 6. The Asite cations in the pyrochlore structures exhibit poor OER activity due to weak *O binding energies (Table S17), so this study focuses solely on Ru active sites. Figure 6A shows the 4-electron OER free energy diagram for a representative $\mathrm{Ru}$ active site on the $\mathrm{Nd}_{2} \mathrm{Ru}_{2} \mathrm{O}_{7}$ pyrochlore and a pyrochlore-like $\mathrm{RuO}_{3}$ structure with fully leached A-site cations. The $\mathrm{Ru}$ site in the $\mathrm{RuO}_{3}$ leached structure shows a distinct shift towards weaker adsorption energies of the OER intermediates, leading to a lower theoretical overpotential of $0.5 \mathrm{~V}$ vs. RHE compared to $0.8 \mathrm{~V}$ vs. RHE for pristine $\mathrm{Nd}_{2} \mathrm{Ru}_{2} \mathrm{O}_{7}$.

We extended the theoretical OER activity analysis for all of the pyrochlore structures investigated in this study with increasing numbers of leached A-site cation layers, as illustrated in Figure 6B. The volcano plot in this figure displays the theoretical OER overpotential for an equivalent $\mathrm{Ru}$ active site in pristine pyrochlores and pyrochlore structures with 1 and 2 layers of leached A-site cations as a function of the $\Delta \mathrm{G}_{\mathrm{O}}-\Delta \mathrm{G}_{\mathrm{OH}}$ binding energy OER descrip- 
tor. The results indicate a distinct shift towards higher OER activity for leached structures compared to the pristine pyrochlores in each case. The fully leached pyrochlore-like $\mathrm{RuO}_{3}$ structure exhibits the highest theoretical OER activity.

We note that the semi-local DFT methods used for theoretical activity predictions in this study are prone to electronic correlation and self-interaction errors. This can lead to inaccuracies in computed electronic properties in non-metallic systems due to excessive delocalization of electron density. ${ }^{78}$ One approach to address this issue is to incorporate a Hubbard- $U$ correction $(\mathrm{DFT}+U)$ that localizes electron density through an effective, rotationally invariant on-site Coulomb repulsion parameter, $U \cdot{ }^{79,80}$ We investigated the sensitivity of our OER activity predictions to the Hubbard- $U$ correction for the most insulating $\mathrm{Y}_{2} \mathrm{Ru}_{2} \mathrm{O}_{7}$ pyrochlore when applied to $4 d$-states of Ru and Y (Figure S31). Our results indicate a systematic shift towards weaker OER intermediate adsorption energies with increasing $U$ values, resulting in higher theoretical OER activity, consistent with previous similar studies for OER. ${ }^{81,82}$ These weakened binding energies and increased theoretical activity predictions reflect the experimentally observed weaker interaction between the $\mathrm{Ru} 4 d$ and $\mathrm{O} 2 p$ orbitals, longer $\mathrm{Ru}-\mathrm{O}$ bond lengths, and higher OER activity of the insulating pyrochlores, particularly $\mathrm{Y}_{2} \mathrm{Ru}_{2} \mathrm{O}_{7}$, compared to $\mathrm{RuO}_{2}$ and $\mathrm{Bi}_{2} \mathrm{Ru}_{2} \mathrm{O}_{7}$. Despite these shifts, the leached pyrochlore structures exhibit weaker binding energies $(>0.5 \mathrm{eV})$ than their pristine counterparts over the entire range of $U$ values considered in this study. These results further underscore the effects of cation leaching on the OER activity and the need for future studies of their electronic structure. While these theoretical OER overpotential predictions offer insight into overall trends in activity with cation leaching, additional factors would need to be considered to directly compare these results with relative experimental onset potentials, including active site density, conductivity, defect concentration, and surface facets. 
A)
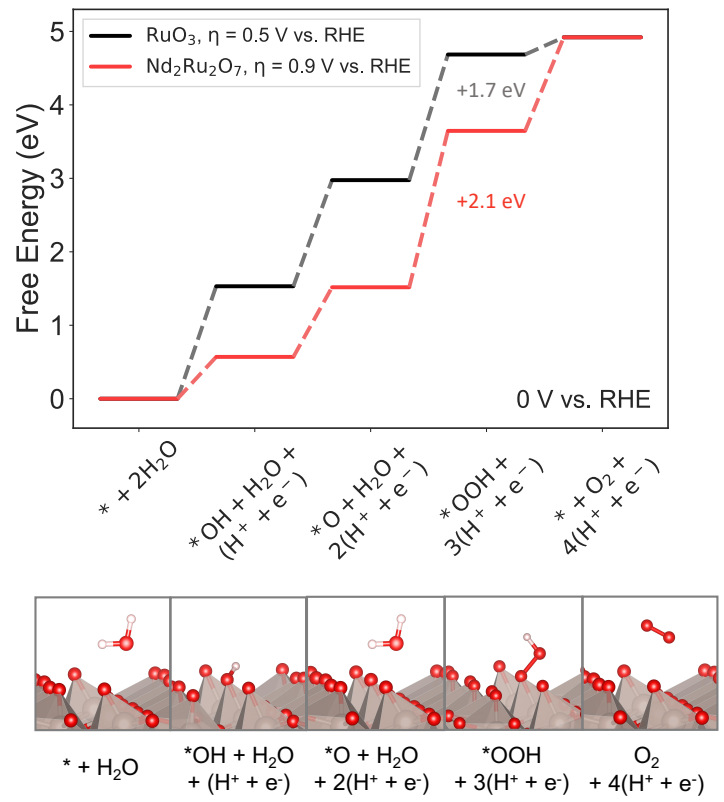

B)
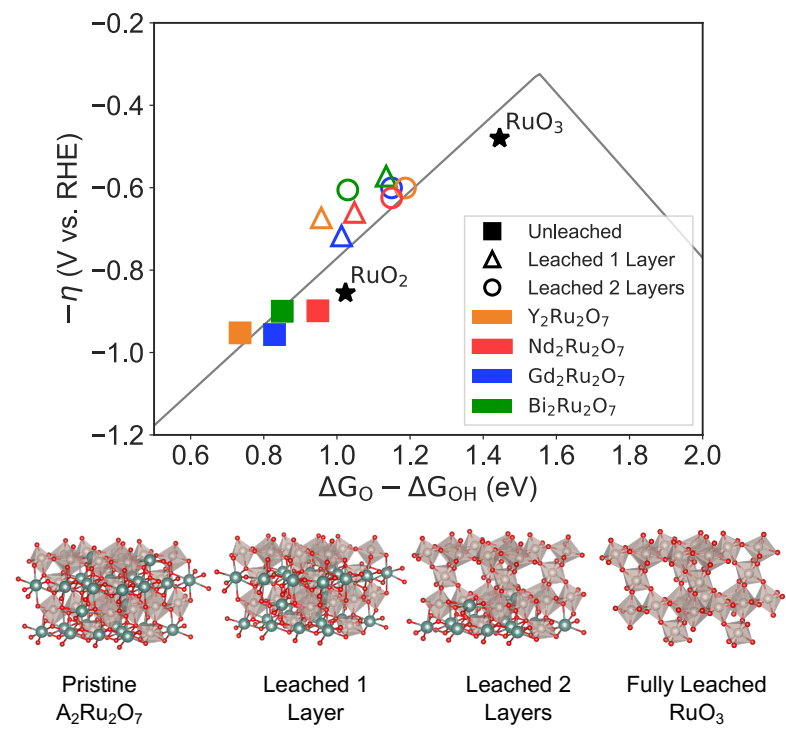

Figure 6: (A) Free energy diagram of a representative $\mathrm{Ru}$ active site on the (111) facet of $\mathrm{Nd}_{2} \mathrm{Ru}_{2} \mathrm{O}_{7}$ versus a fully leached pyrochlore-like $\mathrm{RuO}_{3}$ structure demonstrates a decrease in theoretical OER overpotential. The OER mechanism in the study consists of 4 protoncoupled electron transfer steps to produce $\mathrm{O}_{2}$ from two $\mathrm{H}_{2} \mathrm{O}$ molecules. (B) Activity volcano for pristine and leached pyrochlore phases show an increase in activity with A-site cation leaching. The structures below show the atomistic models of A-leached surfaces. The effect of Hubbard- $U$ correction is shown in Figure S31. 
It is useful to consider the observed activity trends for pristine and leached pyrochlores within the broader context of OER activity predictions for mixed metal oxides. Previous reports indicate that the average $2 p$-state energy of the oxygen atoms within the pyrochlore phase is a useful descriptor for OER activity of various oxide materials. ${ }^{38,57,83,84}$ Other reports have also drawn correlations between the oxidation state of metal atoms and the activity of various oxide phases containing $\mathrm{Ru}$ and Ir. ${ }^{77,85-87}$ We investigated both of these effects in Figure 7 , which displays the $\Delta \mathrm{G}_{\mathrm{O}}-\Delta \mathrm{G}_{\mathrm{OH}}$ binding energy descriptor for leached and pristine pyrochlores as a function of average $\mathrm{O} 2 p$-state energy and $\mathrm{Ru}$ active site oxidation state evaluated via the Bader charge partitioning scheme. The plots show a positive correlation in both cases, and the structures studied within this report fall within the $0.2 \mathrm{eV}$ range of DFT error indicated by the shaded region. These results support the use of these descriptors for OER activity predictions within this material class.

A)

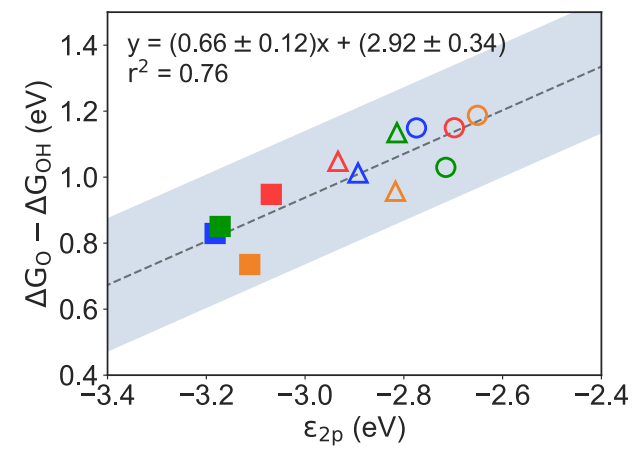

B)

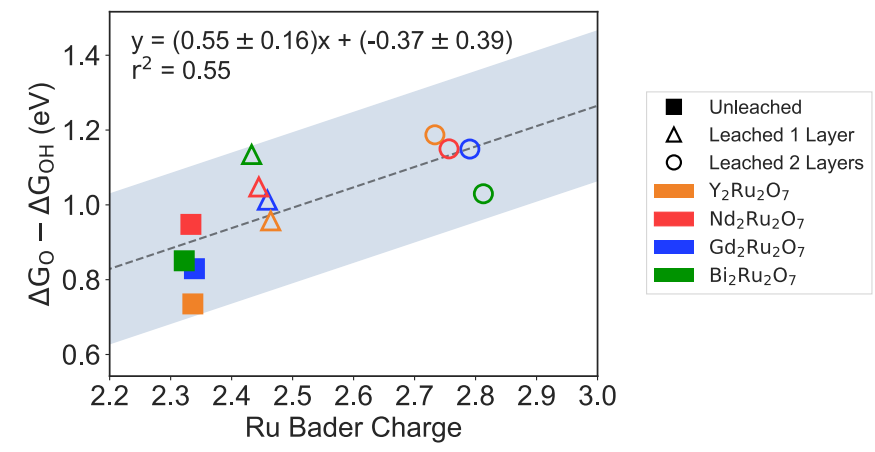

Figure 7: $\Delta \mathrm{G}_{\mathrm{O}}-\Delta \mathrm{G}_{\mathrm{OH}}$ binding energy OER activity descriptor versus (A) average $\mathrm{O} 2 p$ state energy for adsorbed oxygen density of states, and (B) charge on Ru active site. Shaded region represents a $\pm 0.2 \mathrm{eV}$ range for DFT error, and the uncertainty ranges for the slopes and intercepts of each linear fit represent one standard deviation.

Under reaction conditions, the OER activity of the as-synthesized nanoparticulate pyrochlore catalysts likely arises from a combination of active site motifs on various facets of each material. To understand the variation in OER activity for the (111) facet, we used the observed correlation between the average O $2 p$-state energy and the $\Delta \mathrm{G}_{\mathrm{O}}-\Delta \mathrm{G}_{\mathrm{OH}}$ binding 
energy descriptor to consider an ensemble of active sites for each leached and pristine pyrochlore. Based on the linear fit between the $\Delta \mathrm{G}_{\mathrm{O}}-\Delta \mathrm{G}_{\mathrm{OH}}$ binding energy descriptor and the $\mathrm{O} 2 p$-state center in Figure 7A, we find that the optimal O $2 p$-state center corresponding to the peak of the theoretical OER volcano in Figure 6 for these systems is approximately $-2.0 \mathrm{eV}$. This optimal value is indicated by the dashed grey line in Figure 8. Within this figure, the average surface O $2 p$-state center for each structure is represented by bars, while starred markers indicate the maximum value of an ensemble of active sites. The OER activity of these structures most probably arises from a combination of active sites, and those with the maximum $\mathrm{O} 2 p$-state center value will have a higher intrinsic activity and therefore dominate the catalytic activity. Based on our results, both the average and maximum surface O $2 p$-state centers of all of the structures considered herein fall below the optimal value for OER. However, Figure 8 exhibits a clear upward trend in both the average and maximum O $2 p$-state center with A-site cation leaching, indicating an increase in activity with A-site dissolution for all of the pyrochlore structures considered in this study. These results are also in line with the combined CA and ICP-MS results (Figure 3), which suggest a link between OER activity and catalyst stability evidenced by the simultaneous increase in initial CA current density (and TOF) and A-site leaching, especially in the case of $\mathrm{Nd}_{2} \mathrm{Ru}_{2} \mathrm{O}_{7}$. We hypothesize that the larger experimentally observed disparity between A-site and Ru leaching rates in $\mathrm{Nd}_{2} \mathrm{Ru}_{2} \mathrm{O}_{7}$ compared to the other pyrochlores may contribute to a greater density of highly oxidized $\mathrm{Ru}$ active sites, which exhibit favorable OER activity. Collectively, our results further motivate strategies to design active Ru-based OER catalysts by stabilizing highly oxidized $\mathrm{Ru}$ active sites in mixed metal oxides. 


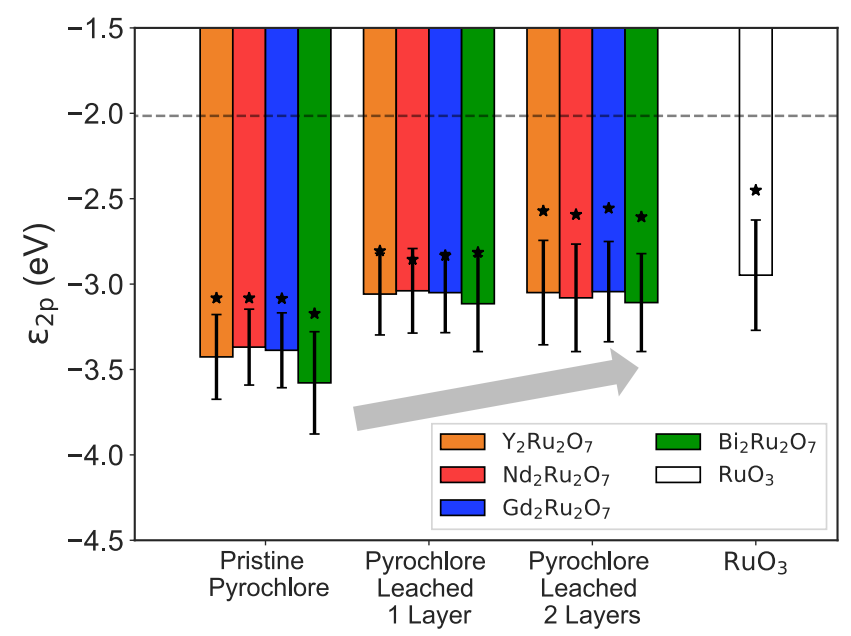

Figure 8: Average calculated O 2p-state energies for the pyrochlore structures and the pyrochlore-like leached $\mathrm{RuO}_{3}$ approaching the extrapolated optimal value of $-2.0 \mathrm{eV}$ for OER activity. Bars represent the average $2 p$-state center values for each surface while stars represent the maximum values. Error bars denote the standard deviation.

\section{Conclusion}

In this work, we have synthesized crystalline $\mathrm{A}_{2} \mathrm{Ru}_{2} \mathrm{O}_{7}(\mathrm{~A}=\mathrm{Y}, \mathrm{Nd}, \mathrm{Gd}, \mathrm{Bi})$ catalysts and have shown that varying the A-site element impacts both the OER activity and stability in acidic electrolyte. Relative to $\mathrm{RuO}_{2}$, we observed enhanced initial OER activity of $\mathrm{A}_{2} \mathrm{Ru}_{2} \mathrm{O}_{7}$ catalysts with longer $\mathrm{Ru}-\mathrm{O}$ bonds and weaker interactions between the $\mathrm{Ru} 4 d$ and $\mathrm{O} 2 p$ orbitals, suggesting weaker OER intermediate adsorption energies. During OER catalysis we experimentally quantified varying A-site and $\mathrm{Ru}$ dissolution rates, which were influenced by the nature of the A-site element. The observed dissolution is consistent with theoretical Pourbaix analysis, indicating that these catalysts are thermodynamically unstable under acidic, oxidizing OER conditions. However, purely thermodynamic predictions and bulk material properties alone do not fully describe the observed differences in dissolution kinetics of different $\mathrm{A}_{2} \mathrm{Ru}_{2} \mathrm{O}_{7}$ compositions. Our combined experimental and theoretical results demonstrate that catalyst dissolution strongly influences OER performance, indicating that 
the relative dissolution rates of $\mathrm{A}$-site and $\mathrm{Ru}$ expose highly oxidized $\mathrm{Ru}$ sites on the catalyst surface. Our theoretical activity predictions show that these leached surface structures exhibit more favorable OER activity than the pristine pyrochlore structures. Future work will aim to experimentally characterize the dynamic catalyst surface properties during OER. Despite the observed dissolution, all $\mathrm{A}_{2} \mathrm{Ru}_{2} \mathrm{O}_{7}$ catalysts studied in this work demonstrated greater experimental activity and stability than a $\mathrm{RuO}_{2}$ standard during OER. These results show the promise of mixed metal oxide structures to stabilize Ru-based OER catalysts without compromising activity and the potential to improve PEM electrolyzer efficiency.

\section{Author Contributions}

M.A.H synthesized, characterized, and performed electrochemical testing of all catalysts. M.A.H., A.G., E.V., J.S., and D.S. collected XAS measurements. A.G. simulated XAS spectra. M.Ben-Naim collected AES maps and SEM images. L.A.K. collected SEM images. Y.L. collected STEM images and STEM-EDS mapping data under the advisement of R.S. A.M.P. performed DFT computations for theoretical predictions and formatted the computational data for the catalysis-hub.org repository. All authors contributed to data analysis and interpretations. M.A.H and A.M.P. prepared this manuscript. M.A.H., A.M.P., J.N., M.B., L.A.K., and T.F.J. formulated the problem and defined the scope. All authors revised this manuscript.

\section{Supporting Information Available}

Catalyst physical properties, as-prepared and post-test characterization, electrochemical activity and stability, example calculations for stability metrics, computational details of DFT simulations, DFT + U sensitivity data, Pourbaix analysis details. 


\section{Acknowledgement}

This research was supported by the U.S. Department of Energy, Office of Science, Office of Basic Energy Sciences, Chemical Sciences, Geosciences, and Biosciences Division, Catalysis Science Program to the SUNCAT Center for Interface Science and Catalysis. Physical characterization of the catalysts in this work was performed at the Stanford Nano Shared Facilities (SNSF), supported by the National Science Foundation under award ECCS-1542152. Juliet Jamtgaard and Arturas Vailionis are acknowledged for their support in XPS and XRD interpretation, respectively. The authors acknowledge the Stanford University Environmental Measurements Facility and Guangchao Li for ICP-MS measurements. Use of Stanford Synchrotron Radiation Lightsource (SSRL), SLAC National Accelerator Laboratory is supported by the US Department of Energy, Office of Science, Office of Basic Energy Science, under Contract DE-AC02-76SF00515. The authors acknowledge the SSRL staff, specifically Dennis Nordlund, Matthew Latimer, and Erik Nelson. M.A.H and A.M.P. acknowledge the support of National Science Foundation Graduate Research Fellowship Program under Grant No. DGE-1656518, and J.K.N acknowledges the support of the Villum Foundation under Grant No. 9455. Any opinions, findings, and conclusions or recommendations expressed in this material are those of the author(s) and do not necessarily reflect the views of the National Science Foundation. The authors would like to acknowledge the use of the computer time allocation for the m2997 allocation at the National Energy Research Scientific Computing Center, a DOE Office of Science User Facility supported by the Office of Science of the U.S. Department of Energy under Contract No. DE-AC02-05CH11231.

\section{References}

(1) Moniz, E. J.; Kenderdine, M. A.; Kizer, A.; Bushman, T.; Canavati, A.; Savitz, S. Optionality, flexibility \& innovation: Pathways for deep decarbonization in California. Energy Futures Initiative. 2019. 
(2) Ruth, M. H2 @ Scale Value Proposition. H2@Scale R\&D Consortium Kickoff Meeting. Chicago, 2018.

(3) Bertuccioli, L.; Chan, A.; Hart, D.; Lehner, F.; Madden, B.; Standen, E. Study on development of water electrolysis in the EU. Fuel Cells and Hydrogen Joint Undertaking 2014, 1-160.

(4) Carmo, M.; Fritz, D. L.; Mergel, J.; Stolten, D. A comprehensive review on PEM water electrolysis. International Journal of Hydrogen Energy 2013, 38, 4901-4934.

(5) Kibsgaard, J.; Chorkendorff, I. Considerations for the scaling-up of water splitting catalysts. Nature Energy 2019, 4, 430-433.

(6) Ayers, K.; Danilovic, N.; Ouimet, R.; Carmo, M.; Pivovar, B.; Bornstein, M. Perspectives on low temperature electrolysis and potential for renewable hydrogen at scale. Annual Review of Chemical and Biomolecular Engineering 2019, 10, 219-239.

(7) McCrory, C. C. L.; Jung, S.; Ferrer, I. M.; Chatman, S. M.; Peters, J. C.; Jaramillo, T. F. Benchmarking Hydrogen Evolving Reaction and Oxygen Evolving Reaction Electrocatalysts for Solar Water Splitting Devices. Journal of the American Chemical Society 2015, 137, 4347-4357.

(8) Moreno-Hernandez, I. A.; MacFarland, C. A.; Read, C. G.; Papadantonakis, K. M.; Brunschwig, B. S.; Lewis, N. S. Crystalline nickel manganese antimonate as a stable water-oxidation catalyst in aqueous 1.0 M H 2 SO 4. Energy \& Environmental Science 2017, 10, 2103-2108.

(9) Danilovic, N.; Subbaraman, R.; Chang, K.-C.; Chang, S. H.; Kang, Y. J.; Snyder, J.; Paulikas, A. P.; Strmcnik, D.; Kim, Y.-T.; Myers, D.; Stamenkovic, V. R.; Markovic, N. M. Activity Stability Trends for the Oxygen Evolution Reaction on Monometallic Oxides in Acidic Environments. The Journal of Physical Chemistry Letters 2014, 5, 2474-2478. 
(10) Lebedev, D.; Povia, M.; Waltar, K.; Abdala, P. M.; Castelli, I. E.; Fabbri, E.; Blanco, M. V.; Fedorov, A.; Copéret, C.; Marzari, N.; Schmidt, T. J. Highly Active and Stable Iridium Pyrochlores for Oxygen Evolution Reaction. Chemistry of Materials 2017, 29, 5182-5191.

(11) Shang, C.; Cao, C.; Yu, D.; Yan, Y.; Lin, Y.; Li, H.; Zheng, T.; Yan, X.; Yu, W.; Zhou, S.; Zeng, J. Electron Correlations Engineer Catalytic Activity of Pyrochlore Iridates for Acidic Water Oxidation. Advanced Materials 2018, 31, 1805104.

(12) Abbott, D. F.; Pittkowski, R. K.; Macounová, K.; Nebel, R.; Marelli, E.; Fabbri, E.; Castelli, I. E.; Krtil, P.; Schmidt, T. J. Design and Synthesis of Ir/Ru Pyrochlore Catalysts for the Oxygen Evolution Reaction Based on Their Bulk Thermodynamic Properties. ACS Applied Materials \& Interfaces 2019, 11, 37748-37760.

(13) Seitz, L. C.; Dickens, C. F.; Nishio, K.; Hikita, Y.; Montoya, J.; Doyle, A.; Kirk, C.; Vojvodic, A.; Hwang, H. Y.; Norskov, J. K.; Jaramillo, T. F. A highly active and stable IrO x /SrIrO 3 catalyst for the oxygen evolution reaction. Science 2016, 353, $1011-1014$.

(14) Gu, X.-K.; Samira, S.; Nikolla, E. Oxygen Sponges for Electrocatalysis: Oxygen Reduction/Evolution on Nonstoichiometric, Mixed Metal Oxides. Chemistry of Materials 2018, 30, 2860-2872.

(15) Vonrüti, N.; Aschauer, U. The role of metastability in enhancing water-oxidation activity. Physical Chemistry Chemical Physics 2019, 21, 24354-24360.

(16) Kim, J.; Shih, P.-C.; Tsao, K.-C.; Pan, Y.-T.; Yin, X.; Sun, C.-J.; Yang, H. HighPerformance Pyrochlore-Type Yttrium Ruthenate Electrocatalyst for Oxygen Evolution Reaction in Acidic Media. Journal of the American Chemical Society 2017, 139, 12076-12083. 
(17) Kuznetsov, D. A.; Naeem, M. A.; Kumar, P. V.; Abdala, P. M.; Fedorov, A.; Müller, C. R. Tailoring Lattice Oxygen Binding in Ruthenium Pyrochlores to Enhance Oxygen Evolution Activity. Journal of the American Chemical Society 2020, 142, 7883-7888.

(18) Retuerto, M.; Pascual, L.; Calle-Vallejo, F.; Ferrer, P.; Gianolio, D.; Pereira, A. G.; García, .; Torrero, J.; Fernández-Díaz, M. T.; Bencok, P.; Peña, M. A.; Fierro, J. L. G.; Rojas, S. Na-doped ruthenium perovskite electrocatalysts with improved oxygen evolution activity and durability in acidic media. Nature Communications 2019, 10, 2041.

(19) Parrondo, J.; George, M.; Capuano, C.; Ayers, K. E.; Ramani, V. Pyrochlore electrocatalysts for efficient alkaline water electrolysis. Journal of Materials Chemistry A 2015, 3, 10819-10828.

(20) Subramanian, M.; Aravamudan, G.; Subba Rao, G. Oxide pyrochlores A review. Progress in Solid State Chemistry 1983, 15, 55-143.

(21) Suntivich, J.; Hong, W. T.; Lee, Y.-L.; Rondinelli, J. M.; Yang, W.; Goodenough, J. B.; Dabrowski, B.; Freeland, J. W.; Shao-Horn, Y. Estimating Hybridization of Transition Metal and Oxygen States in Perovskites from O K -edge X-ray Absorption Spectroscopy. The Journal of Physical Chemistry C 2014, 118, 1856-1863.

(22) Miao, X.; Zhang, L.; Wu, L.; Hu, Z.; Shi, L.; Zhou, S. Quadruple perovskite ruthenate as a highly efficient catalyst for acidic water oxidation. Nature Communications 2019, 10,3809 .

(23) Kim, J.; Shih, P.; Qin, Y.; AlBardan, Z.; Sun, C.; Yang, H. A Porous Pyrochlore Y 2 $[\mathrm{Ru} 1.6 \mathrm{Y} 0.4] \mathrm{O} 7 \delta$ Electrocatalyst for Enhanced Performance towards the Oxygen Evolution Reaction in Acidic Media. Angewandte Chemie 2018, 130, 14073-14077. 
(24) Morgan, D. J. Resolving ruthenium: XPS studies of common ruthenium materials. Surface and Interface Analysis 2015, 47, 1072-1079.

(25) Ravel, B.; Newville, M. ATHENA , ARTEMIS , HEPHAESTUS : data analysis for Xray absorption spectroscopy using IFEFFIT. Journal of Synchrotron Radiation 2005, 12, 537-541.

(26) Alia, S. M.; Anderson, G. C. Iridium Oxygen Evolution Activity and Durability Baselines in Rotating Disk Electrode Half-Cells. Journal of The Electrochemical Society 2019, 166, F282-F294.

(27) Geiger, S.; Kasian, O.; Mingers, A. M.; Nicley, S. S.; Haenen, K.; Mayrhofer, K. J. J.; Cherevko, S. Catalyst Stability Benchmarking for the Oxygen Evolution Reaction: The Importance of Backing Electrode Material and Dissolution in Accelerated Aging Studies. ChemSusChem 2017, 10, 4140-4143.

(28) Yi, Y.; Weinberg, G.; Prenzel, M.; Greiner, M.; Heumann, S.; Becker, S.; Schlögl, R. Electrochemical corrosion of a glassy carbon electrode. Catalysis Today 2017, 295, $32-40$.

(29) Gibbons, B. M.; Wette, M.; Stevens, M. B.; Davis, R. C.; Siahrostami, S.; Kreider, M.; Mehta, A.; Higgins, D. C.; Clemens, B. M.; Jaramillo, T. F. In Situ X-Ray Absorption Spectroscopy Disentangles the Roles of Copper and Silver in a Bimetallic Catalyst for the Oxygen Reduction Reaction. Chemistry of Materials 2020, 32, 1819-1827.

(30) Perdew, J. P.; Burke, K.; Ernzerhof, M. Generalized Gradient Approximation Made Simple. Physical Review Letters 1996, 77, 3865-3868.

(31) Kresse, G.; Hafner, J. Ab initio molecular dynamics for liquid metals. Physical Review $B$ 1993, 47, 558-561. 
(32) Kresse, G.; Furthmüller, J. Efficiency of ab-initio total energy calculations for metals and semiconductors using a plane-wave basis set. Computational Materials Science 1996, 6, 15-50.

(33) Kresse, G.; Furthmüller, J. Efficient iterative schemes for ab initio total-energy calculations using a plane-wave basis set. Physical Review B 1996, 54, 11169-11186.

(34) Kresse, G.; Joubert, D. From ultrasoft pseudopotentials to the projector augmentedwave method. Physical Review B 1999, 59, 1758-1775.

(35) Pack, J. D.; Monkhorst, H. J. "Special points for Brillouin-zone integrations"a reply. Physical Review B 1977, 16, 1748-1749.

(36) Ong, S. P.; Richards, W. D.; Jain, A.; Hautier, G.; Kocher, M.; Cholia, S.; Gunter, D.; Chevrier, V. L.; Persson, K. A.; Ceder, G. Python Materials Genomics (pymatgen): A robust, open-source python library for materials analysis. Computational Materials Science 2013, 68, 314-319.

(37) Winther, K. T.; Hoffmann, M. J.; Boes, J. R.; Mamun, O.; Bajdich, M.; Bligaard, T. Catalysis-Hub.org, an open electronic structure database for surface reactions. Scientific Data 2019, 6, 75 .

(38) Dickens, C. F.; Montoya, J. H.; Kulkarni, A. R.; Bajdich, M.; Nørskov, J. K. An electronic structure descriptor for oxygen reactivity at metal and metal-oxide surfaces. Surface Science 2019, 681, 122-129.

(39) Tang, W.; Sanville, E.; Henkelman, G. A grid-based Bader analysis algorithm without lattice bias. Journal of Physics: Condensed Matter 2009, 21, 84204.

(40) Ishii, F.; Oguchi, T. Electronic Band Structure of the Pyrochlore Ruthenium Oxides A 2 Ru 2 O 7 ( A = B i, Tl and Y). Journal of the Physical Society of Japan 2000,69, $526-531$. 
(41) Shannon, R. D. Revised Effective Ionic Radii and Systematic Studies of Interatomie Distances in Halides and Chaleogenides. Acta Crystallographica Section A Foundations and Advances 1976, 32, 751-767.

(42) Kennedy, B.; Vogt, T. Structural and Bonding Trends in Ruthenium Pyrochlores. Journal of Solid State Chemistry 1996, 126, 261-270.

(43) Sleight, A. W.; Ramirez, A. P. Disappearance of the metal-insulator transition in iridate pyrochlores on approaching the ideal R 2 Ir 2 O 7 stoichiometry. Solid State Communications 2018, 275, 12-15.

(44) Kanno, R.; Takeda, Y.; Yamamoto, T.; Kawamoto, Y.; Yamamoto, O. Crystal Structure and Electrical Properties of the Pyrochlore Ruthenate Bi2-xYxRu2O7. Journal of Solid State Chemistry 1993, 102, 106-114.

(45) Kobayashi, H.; Kanno, R.; Kawamoto, Y.; Kamiyama, T.; Izumi, F.; Sleight, A. Synthesis, Crystal Structure, and Electrical Properties of the Pyrochlores Pb2-xLnxRu2O7$\mathrm{y}(\mathrm{Ln}=\mathrm{Nd}, \mathrm{Gd})$. Journal of Solid State Chemistry 1995, 114, 15-23.

(46) Hong, W. T.; Risch, M.; Stoerzinger, K. A.; Grimaud, A.; Suntivich, J.; Shao-Horn, Y. Toward the rational design of non-precious transition metal oxides for oxygen electrocatalysis. Energy \& Environmental Science 2015, 8, 1404-1427.

(47) Schmidt, M.; Cummins, T. R.; Bürk, M.; Lu, D. H.; Nücker, N.; Schuppler, S.; Lichtenberg, F. Nature of the electronic states in the layered perovskite noncuprate superconductor Sr2RuO4. Physical Review B 1996, 53, R14761-R14764.

(48) Kang, S.; Tseng, Y.; Kim, B. H.; Yun, S.; Sohn, B.; Kim, B.; McNally, D.; Paris, E.; Kim, C. H.; Kim, C.; Noh, T. W.; Ishihara, S.; Schmitt, T.; Park, J.-G. Orbital-selective confinement effect of Ru 4d orbitals in SrRuO3 ultrathin film. Physical Review B 2019, 99, 045113. 
(49) de Groot, F. M. F.; Grioni, M.; Fuggle, J. C.; Ghijsen, J.; Sawatzky, G. A.; Petersen, H. Oxygen 1 s x-ray-absorption edges of transition-metal oxides. Physical Review B 1989, $40,5715-5723$.

(50) Noh, H.-J.; Kim, B. J.; Oh, S.-J.; Park, J.-H.; Lin, H.-J.; Chen, C. T.; Lee, Y. S.; Yamaura, K.; Takayama-Muromachi, E. Comparative study of the electronic structures of SrMO $3(\mathrm{M}=\mathrm{Ti}, \mathrm{V}, \mathrm{Mn}, \mathrm{Fe}$, and $\mathrm{Co} ; \mathrm{M}=\mathrm{Zr}, \mathrm{Mo}, \mathrm{Ru}$, and Rh) by O 1s x-ray absorption spectroscopy. Journal of Physics: Condensed Matter 2008, 20, 485208.

(51) Hu, Y.-Y.; Liu, Z.; Nam, K.-W.; Borkiewicz, O. J.; Cheng, J.; Hua, X.; Dunstan, M. T.; Yu, X.; Wiaderek, K. M.; Du, L.-S.; Chapman, K. W.; Chupas, P. J.; Yang, X.-Q.; Grey, C. P. Origin of additional capacities in metal oxide lithium-ion battery electrodes. Nature Materials 2013, 12, 1130-1136.

(52) Kim, H.-D.; Noh, H.-J.; Kim, K. H.; Oh, S.-J. Core-Level X-Ray Photoemission Satellites in Ruthenates: A New Mechanism Revealing The Mott Transition. Physical Review Letters 2004, 93, 126404.

(53) Yu, Y. et al. Revealing Electronic Signatures of Lattice Oxygen Redox in Lithium Ruthenates and Implications for High-Energy Li-Ion Battery Material Designs. Chemistry of Materials 2019, 31, 7864-7876.

(54) Li, L.; Kennedy, B. J. Structural and Electronic Properties of the Ru Pyrochlores Bi 2- y Yb y Ru 2 O 7- $\delta$. Chemistry of Materials 2003, 15, 4060-4067.

(55) Field, M.; Kennedy, B.; Hunter, B. Structural Studies of the Metal Nonmetal Transition in Ru Pyrochlores. Journal of Solid State Chemistry 2000, 151, 25-30.

(56) Hodnik, N.; Jovanovič, P.; Pavlišič, A.; Jozinović, B.; Zorko, M.; Bele, M.; Šelih, V. S.; Šala, M.; Hočevar, S.; Gaberšček, M. New Insights into Corrosion of Ruthenium and Ruthenium Oxide Nanoparticles in Acidic Media. The Journal of Physical Chemistry C 2015, 119, 10140-10147. 
(57) Zagalskaya, A.; Alexandrov, V. Role of Defects in the Interplay between Adsorbate Evolving and Lattice Oxygen Mechanisms of the Oxygen Evolution Reaction in RuO 2 and IrO 2. ACS Catalysis 2020, 10, 3650-3657.

(58) Cherevko, S.; Geiger, S.; Kasian, O.; Kulyk, N.; Grote, J.-P.; Savan, A.; Shrestha, B. R.; Merzlikin, S.; Breitbach, B.; Ludwig, A.; Mayrhofer, K. J. Oxygen and hydrogen evolution reactions on $\mathrm{Ru}, \mathrm{RuO} 2$, $\mathrm{Ir}$, and $\mathrm{IrO} 2$ thin film electrodes in acidic and alkaline electrolytes: A comparative study on activity and stability. Catalysis Today 2016, 262, $170-180$.

(59) Roy, C.; Rao, R. R.; Stoerzinger, K. A.; Hwang, J.; Rossmeisl, J.; Chorkendorff, I.; Shao-Horn, Y.; Stephens, I. E. L. Trends in Activity and Dissolution on RuO 2 under Oxygen Evolution Conditions: Particles versus Well-Defined Extended Surfaces. ACS Energy Letters 2018, 3, 2045-2051.

(60) Geiger, S.; Kasian, O.; Ledendecker, M.; Pizzutilo, E.; Mingers, A. M.; Fu, W. T.; Diaz-Morales, O.; Li, Z.; Oellers, T.; Fruchter, L.; Ludwig, A.; Mayrhofer, K. J. J.; Koper, M. T. M.; Cherevko, S. The stability number as a metric for electrocatalyst stability benchmarking. Nature Catalysis 2018, 1, 508-515.

(61) McCafferty, E. Introduction to Corrosion Science; Springer New York: New York, NY, 2010; pp 1-575.

(62) Pourbaix, M. Applications of electrochemistry in corrosion science and in practice. Corrosion Science 1974, 14, 25-82.

(63) Bajdich, M.; García-Mota, M.; Vojvodic, A.; Nørskov, J. K.; Bell, A. T. Theoretical Investigation of the Activity of Cobalt Oxides for the Electrochemical Oxidation of Water. Journal of the American Chemical Society 2013, 135, 13521-13530.

(64) Patel, A. M.; Nørskov, J. K.; Persson, K. A.; Montoya, J. H. Efficient Pourbaix diagrams 
of many-element compounds. Physical Chemistry Chemical Physics 2019, 21, 2532325327.

(65) Jain, A.; Ong, S. P.; Hautier, G.; Chen, W.; Richards, W. D.; Dacek, S.; Cholia, S.; Gunter, D.; Skinner, D.; Ceder, G.; Persson, K. A. Commentary: The Materials Project: A materials genome approach to accelerating materials innovation. APL Materials 2013, 1, 011002.

(66) Belsky, A.; Hellenbrandt, M.; Karen, V. L.; Luksch, P. New developments in the Inorganic Crystal Structure Database (ICSD): accessibility in support of materials research and design. Acta Crystallographica Section B Structural Science 2002, 58, 364-369.

(67) Singh, A. K.; Zhou, L.; Shinde, A.; Suram, S. K.; Montoya, J. H.; Winston, D.; Gregoire, J. M.; Persson, K. A. Electrochemical Stability of Metastable Materials. Chemistry of Materials 2017, 29, 10159-10167.

(68) Kötz, R.; Stucki, S.; Scherson, D.; Kolb, D. In-situ identification of RuO4 as the corrosion product during oxygen evolution on ruthenium in acid media. Journal of Electroanalytical Chemistry and Interfacial Electrochemistry 1984, 172, 211-219.

(69) Cherevko, S. Stability and dissolution of electrocatalysts: Building the bridge between model and real world systems. Current Opinion in Electrochemistry 2018, 8, 118-125.

(70) Fabbri, E.; Habereder, A.; Waltar, K.; Kötz, R.; Schmidt, T. J. Developments and perspectives of oxide-based catalysts for the oxygen evolution reaction. Catal. Sci. Technol. 2014, 4, 3800-3821.

(71) Spöri, C.; Kwan, J. T. H.; Bonakdarpour, A.; Wilkinson, D. P.; Strasser, P. The Stability Challenges of Oxygen Evolving Catalysts: Towards a Common Fundamental Understanding and Mitigation of Catalyst Degradation. Angewandte Chemie International Edition 2017, 56, 5994-6021. 
(72) Dickens, C. F.; Kirk, C.; Nørskov, J. K. Insights into the Electrochemical Oxygen Evolution Reaction with ab Initio Calculations and Microkinetic Modeling: Beyond the Limiting Potential Volcano. The Journal of Physical Chemistry C 2019, 123, 1896018977.

(73) Soriano-López, J.; Schmitt, W.; García-Melchor, M. Computational modelling of water oxidation catalysts. Current Opinion in Electrochemistry 2018, 7, 22-30.

(74) Nørskov, J. K.; Rossmeisl, J.; Logadottir, A.; Lindqvist, L.; Kitchin, J. R.; Bligaard, T.; Jónsson, H. Origin of the Overpotential for Oxygen Reduction at a Fuel-Cell Cathode. The Journal of Physical Chemistry B 2004, 108, 17886-17892.

(75) Rao, R. R. et al. Towards identifying the active sites on RuO 2 (110) in catalyzing oxygen evolution. Energy \&3 Environmental Science 2017, 10, 2626-2637.

(76) Rossmeisl, J.; Qu, Z.-W.; Zhu, H.; Kroes, G.-J.; Nørskov, J. Electrolysis of water on oxide surfaces. Journal of Electroanalytical Chemistry 2007, 607, 83-89.

(77) Rao, R. R.; Kolb, M. J.; Giordano, L.; Pedersen, A. F.; Katayama, Y.; Hwang, J.; Mehta, A.; You, H.; Lunger, J. R.; Zhou, H.; Halck, N. B.; Vegge, T.; Chorkendorff, I.; Stephens, I. E. L.; Shao-Horn, Y. Operando identification of site-dependent water oxidation activity on ruthenium dioxide single-crystal surfaces. Nature Catalysis 2020, 3 , $516-525$.

(78) Seidl, A.; Görling, A.; Vogl, P.; Majewski, J. A.; Levy, M. Generalized Kohn-Sham schemes and the band-gap problem. Physical Review B 1996, 53, 3764-3774.

(79) Dudarev, S. L.; Botton, G. A.; Savrasov, S. Y.; Humphreys, C. J.; Sutton, A. P. Electron-energy-loss spectra and the structural stability of nickel oxide: An LSDA+U study. Physical Review B 1998, 57, 1505-1509. 
(80) Bengone, O.; Alouani, M.; Blöchl, P.; Hugel, J. Implementation of the projector augmented-wave LDA+U method: Application to the electronic structure of NiO. Physical Review B 2000, 62, 16392-16401.

(81) García-Mota, M.; Bajdich, M.; Viswanathan, V.; Vojvodic, A.; Bell, A. T.; Nørskov, J. K. Importance of Correlation in Determining Electrocatalytic Oxygen Evolution Activity on Cobalt Oxides. The Journal of Physical Chemistry C 2012, 116, 21077-21082.

(82) Xu, Z.; Rossmeisl, J.; Kitchin, J. R. A Linear Response DFT+ U Study of Trends in the Oxygen Evolution Activity of Transition Metal Rutile Dioxides. The Journal of Physical Chemistry C 2015, 119, 4827-4833.

(83) Grimaud, A.; May, K. J.; Carlton, C. E.; Lee, Y.-L.; Risch, M.; Hong, W. T.; Zhou, J.; Shao-Horn, Y. Double perovskites as a family of highly active catalysts for oxygen evolution in alkaline solution. Nature Communications 2013, 4, 2439.

(84) Grimaud, A.; Diaz-Morales, O.; Han, B.; Hong, W. T.; Lee, Y.-L.; Giordano, L.; Stoerzinger, K. A.; Koper, M. T. M.; Shao-Horn, Y. Activating lattice oxygen redox reactions in metal oxides to catalyse oxygen evolution. Nature Chemistry 2017, 9, 457-465.

(85) Strickler, A. L.; Flores, R. A.; King, L. A.; Nørskov, J. K.; Bajdich, M.; Jaramillo, T. F. Systematic Investigation of Iridium-Based Bimetallic Thin Film Catalysts for the Oxygen Evolution Reaction in Acidic Media. ACS Applied Materials \& Interfaces 2019, 11, 34059-34066.

(86) Klyukin, K.; Zagalskaya, A.; Alexandrov, V. Role of Dissolution Intermediates in Promoting Oxygen Evolution Reaction at RuO 2 (110) Surface. The Journal of Physical Chemistry C 2019, 123, 22151-22157.

(87) Huang, X.; Wang, J.; Tao, H. B.; Tian, H.; Xu, H. An essential descriptor for the 
oxygen evolution reaction on reducible metal oxide surfaces. Chemical Science 2019, 10, 3340-3345. 


\section{Graphical TOC Entry}

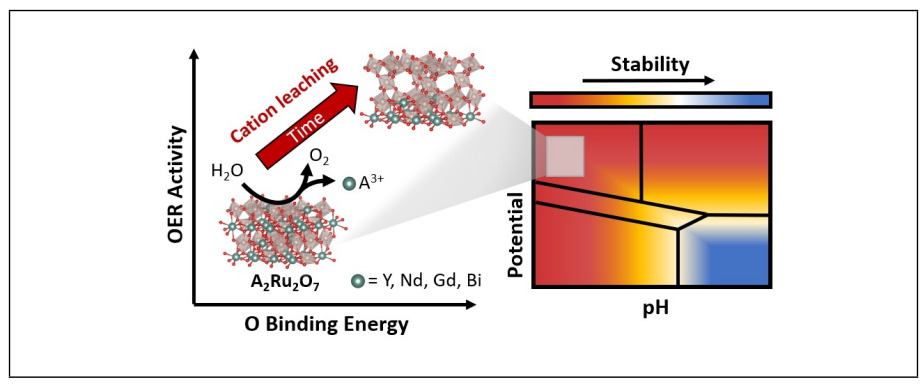

